\title{
Planck early results. XXI. Properties of the interstellar medium in the Galactic plane ${ }^{\star}$
}

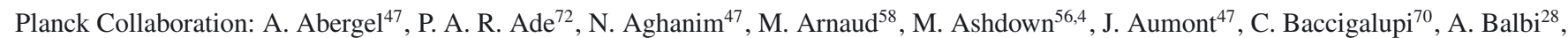
A. J. Banday ${ }^{76,763}$, R. B. Barreiro ${ }^{53}$, J. G. Bartlett ${ }^{3,54}$, E. Battaner ${ }^{78}$, K. Benabed $^{48}$, A. Benoît ${ }^{46}$, J.-P. Bernard ${ }^{76,7}$, M. Bersanelli ${ }^{25,41}$, R. Bhatia $^{5}$, J. J. Bock ${ }^{54,8}$, A. Bonaldi ${ }^{37}$, J. R. Bond ${ }^{6}$, J. Borrill ${ }^{62,73}$, F. R. Bouchet ${ }^{48}$, F. Boulanger ${ }^{47}$, M. Bucher ${ }^{3}$, C. Burigana ${ }^{40}$, P. Cabella ${ }^{28}$,

J.-F. Cardoso ${ }^{59,3,48}$, A. Catalano ${ }^{3,57}$, L. Cayón ${ }^{18}$, A. Challinor ${ }^{50,56,10}$, A. Chamballu ${ }^{44}$, L.-Y Chiang ${ }^{49}$, C. Chiang ${ }^{17}$, P. R. Christensen ${ }^{67,29}$, S. Colombi ${ }^{48}$, F. Couchot ${ }^{61}$, A. Coulais ${ }^{57}$, B. P. Crill ${ }^{54,68}$, F. Cuttaia ${ }^{40}$, T. M. Dame ${ }^{34}$, L. Danese ${ }^{70}$, R. D. Davies ${ }^{55}$, R. J. Davis ${ }^{55}$, P. de Bernardis ${ }^{24}$, G. de Gasperis ${ }^{28}$, A. de Rosa ${ }^{40}$, G. de Zotti ${ }^{37,70}$, J. Delabrouille ${ }^{3}$, J.-M. Delouis ${ }^{48}$, F.-X. Désert ${ }^{43}$, C. Dickinson ${ }^{55}$, S. Donzelli ${ }^{41,51}$, O. Doré ${ }^{54,8}$, U. Dörl ${ }^{63}$, M. Douspis ${ }^{47}$, X. Dupac ${ }^{32}$, G. Efstathiou ${ }^{50}$, T. A. Enßlin ${ }^{63}$, F. Finelli ${ }^{40}$, O. Forni ${ }^{76,7}$, M. Frailis ${ }^{39}$, E. Franceschi ${ }^{40}$, S. Galeotta ${ }^{39}$,

K. Ganga ${ }^{3,45}$, M. Giard ${ }^{76,7}$, G. Giardino ${ }^{33}$, Y. Giraud-Héraud ${ }^{3}$, J. González-Nuevo ${ }^{70}$, K. M. Górski ${ }^{54,80}$, S. Gratton ${ }^{56,50}$, A. Gregorio ${ }^{26}$,

I. A. Grenier ${ }^{58}$, A. Gruppuso ${ }^{40}$, F. K. Hansen ${ }^{51}$, D. Harrison ${ }^{50,56}$, S. Henrot-Versillé ${ }^{61}$, D. Herranz ${ }^{53}$, S. R. Hildebrandt ${ }^{8,60,52}$, E. Hivon ${ }^{48}$,

M. Hobson ${ }^{4}$, W. A. Holmes ${ }^{54}$, W. Hovest ${ }^{63}$, R. J. Hoyland ${ }^{52}$, K. M. Huffenberger ${ }^{79}$, T. R. Jaffe ${ }^{76,7}$, A. H. Jaffe ${ }^{44}$, W. C. Jones ${ }^{17}$, M. Juvela ${ }^{16}$,

E. Keihänen ${ }^{16}$, R. Keskitalo ${ }^{54,16}$, T. S. Kisner ${ }^{62}$, R. Kneissl ${ }^{31,5}$, L. Knox ${ }^{20}$, H. Kurki-Suonio ${ }^{16,35}$, G. Lagache ${ }^{47}$, A. Lähteenmäki ${ }^{1,35}$,

J.-M. Lamarre ${ }^{57}$, A. Lasenby ${ }^{4,56}$, R. J. Laureijs ${ }^{33}$, C. R. Lawrence ${ }^{54}$, S. Leach ${ }^{70}$, R. Leonardi ${ }^{32,33,21}$, C. Leroy ${ }^{47,76,7}$, P. B. Lilje ${ }^{51,9}$,

M. Linden-Vørnle ${ }^{12}$, M. López-Caniego ${ }^{53}$, P. M. Lubin ${ }^{21}$, J. F. Macías-Pérez ${ }^{60}$, C. J. MacTavish ${ }^{56}$, B. Maffei ${ }^{55}$, N. Mandolesi ${ }^{40}$, R. Mann ${ }^{71}$,

M. Maris ${ }^{39}$, D. J. Marshall ${ }^{76,7}$, E. Martínez-González ${ }^{53}$, S. Masi ${ }^{24}$, S. Matarrese ${ }^{23}$, F. Matthai ${ }^{63}$, P. Mazzotta ${ }^{28}$, P. McGehee ${ }^{45}$, P. R. Meinhold ${ }^{21}$,

A. Melchiorri ${ }^{24}$, L. Mendes ${ }^{32}$, A. Mennella ${ }^{25,39}$, M.-A. Miville-Deschênes ${ }^{47,6}$, A. Moneti ${ }^{48}$, L. Montier ${ }^{76,7}$, G. Morgante ${ }^{40}$, D. Mortlock ${ }^{44}$,

D. Munshi ${ }^{72,50}$, A. Murphy ${ }^{66}$, P. Naselsky ${ }^{67,29}$, P. Natoli ${ }^{27,2,40}$, C. B. Netterfield ${ }^{14}$, H. U. Nørgaard-Nielsen ${ }^{12}$, F. Noviello ${ }^{47}$, D. Novikov $^{44}$,

I. Novikov ${ }^{67}$, S. Osborne ${ }^{75}$, F. Pajot ${ }^{47}$, R. Paladini ${ }^{74,8}$, F. Pasian ${ }^{39}$, G. Patanchon ${ }^{3}$, O. Perdereau ${ }^{61}$, L. Perotto ${ }^{60}$, F. Perrotta ${ }^{70}$, F. Piacentini ${ }^{24}$,

M. Piat ${ }^{3}$, S. Plaszczynski ${ }^{61}$, E. Pointecouteau ${ }^{76,7}$, G. Polenta ${ }^{2,38}$, N. Ponthieu ${ }^{47}$, T. Poutanen ${ }^{35,16,1}$, G. Prézeau ${ }^{8,54}$, S. Prunet ${ }^{48}$, J.-L. Puget ${ }^{47}$,

J. P. Rachen ${ }^{63}$, W. T. Reach ${ }^{77}$, R. Rebolo ${ }^{52,30}$, W. Reich ${ }^{64}$, C. Renault ${ }^{60}$, S. Ricciardi ${ }^{40}$, T. Riller ${ }^{63}$, I. Ristorcelli ${ }^{76,7}$, G. Rocha $^{54,8}$, C. Rosset $^{3}$,

J. A. Rubiño-Martín ${ }^{52,30}$, B. Rusholme ${ }^{45}$, M. Sandri ${ }^{40}$, D. Santos ${ }^{60}$, G. Savini ${ }^{69}$, D. Scott ${ }^{15}$, M. D. Seiffert ${ }^{54,8}$, P. Shellard ${ }^{10}$, G. F. Smoot ${ }^{19,62,3}$,

J.-L. Starck ${ }^{58,11}$, F. Stivoli ${ }^{42}$, V. Stolyarov ${ }^{4}$, R. Stompor ${ }^{3}$, R. Sudiwala ${ }^{72}$, J.-F. Sygnet ${ }^{48}$, J. A. Tauber ${ }^{33}$, L. Terenzi ${ }^{40}$, L. Toffolatti ${ }^{13}$,

M. Tomasi ${ }^{25,41}$, J.-P. Torre ${ }^{47}$, M. Tristram ${ }^{61}$, J. Tuovinen ${ }^{65}$, G. Umana ${ }^{36}$, L. Valenziano ${ }^{40}$, J. Varis ${ }^{65}$, P. Vielva ${ }^{53}$, F. Villa ${ }^{40}$, N. Vittorio $^{28}$, L. A. Wade ${ }^{54}$, B. D. Wandelt ${ }^{48,22}$, A. Wilkinson ${ }^{55}$, N. Ysard ${ }^{16}$, D. Yvon ${ }^{11}$, A. Zacchei ${ }^{39}$, and A. Zonca ${ }^{21}$

(Affiliations can be found after the references)

Received 7 January 2011 / Accepted 9 June 2011

\begin{abstract}
Planck has observed the entire sky from $30 \mathrm{GHz}$ to $857 \mathrm{GHz}$. The observed foreground emission contains contributions from different phases of the interstellar medium (ISM). We have separated the observed Galactic emission into the different gaseous components (atomic, molecular and ionised) in each of a number of Galactocentric rings. This technique provides the necessary information to study dust properties (emissivity, temperature, etc.), as well as other emission mechanisms as a function of Galactic radius. Templates are created for various Galactocentric radii using velocity information from atomic (neutral hydrogen) and molecular $\left({ }^{12} \mathrm{CO}\right)$ observations. The ionised template is assumed to be traced by free-free emission as observed by WMAP, while $408 \mathrm{MHz}$ emission is used to trace the synchrotron component. Gas emission not traced by the above templates, namely "dark gas", as evidenced using Planck data, is included as an additional template, the first time such a component has been used in this way. These templates are then correlated with each of the Planck frequency bands, as well as with higher frequency data from IRAS and DIRBE along with radio data at $1.4 \mathrm{GHz}$. The emission per column density of the gas templates allows us to create distinct spectral energy distributions (SEDs) per Galactocentric ring and in each of the gaseous tracers from $1.4 \mathrm{GHz}$ to $25 \mathrm{THz}$ (12 $\mu \mathrm{m})$. The resulting SEDs allow us to explore the contribution of various emission mechanisms to the Planck signal. Apart from the thermal dust and free-free emission, we have probed the Galaxy for anomalous (e.g., spinning) dust as well as synchrotron emission. We find the dust opacity in the solar neighbourhood, $\tau / N_{\mathrm{H}}=0.92 \pm 0.05 \times 10^{-25} \mathrm{~cm}^{2}$ at $250 \mu \mathrm{m}$, with no significant variation with Galactic radius, even though the dust temperature is seen to vary from over $25 \mathrm{~K}$ to under $14 \mathrm{~K}$. Furthermore, we show that anomalous dust emission is present in the atomic, molecular and dark gas phases throughout the Galactic disk. Anomalous emission is not clearly detected in the ionised phase, as free-free emission is seen to dominate. The derived dust propeties associated with the dark gas phase are derived but do not allow us to reveal the nature of this phase. For all environments, the anomalous emission is consistent with rotation from polycyclic aromatic hydrocarbons (PAHs) and, according to our simple model, accounts for (25 \pm 5 ) $\%$ (statistical) of the total emission at $30 \mathrm{GHz}$.
\end{abstract}

Key words. ISM: general - Galaxy: general - radio continuum: ISM - submillimeter: ISM - infrared: ISM - radiation mechanisms: general

^ Corresponding author: D. J. Marshall, e-mail: douglas.marshall@irap.omp.eu 


\section{Introduction}

In order to understand our own Galaxy, it is necessary to explore Galactic plane $\left(|b| \lesssim 10^{\circ}\right)$, where we are able to observe the emission coming from a large range of distances. However the observed emission is the sum of a large number of line-of-sight components, often probing very different environments.

Several previous studies (Bloemen et al. 1986; Bloemen et al. 1990; Giard et al. 1994; Sodroski et al. 1997; Paladini et al. 2007) have separated observed integrated emission into a number of Galactocentric radii in order to study its properties as a function of Galactic position and in different phases of the interstellar gas (e.g., atomic, molecular and ionised). The radial velocity of the gas is used to separate the Galactic gas emission into a number of Galactocentric rings and then the spectral energy distribution (SED) of each ring/gas phase is fitted with a physical model of dust and gas emissions. These methods have been used successfully in previous studies to map out basic properties of the ISM throughout the Galaxy.

For instance, Giard et al. (1994) demonstrated that polycyclic aromatic hydrocarbons (PAHs) are a ubiquitous component of the ISM, and Bloemen et al. (1990) showed that the dust temperature decreases with distance to the Galactic Centre in a way which is fully consistent with an exponential decrease of the interstellar radiation field (ISRF) in the stellar disk. Sodroski et al. (1997) suggested that the abundance of large dust grains within each gas phase exhibits a gradient that is equivalent, within the uncertainties, to the metallicity gradient in the Galactic disk. Paladini et al. (2007) showed that the dust in molecular clouds appears to be heated in a significant way by young massive stars still embedded in their parent clouds.

With the advent of the Planck satellite ${ }^{1}$, it is now possible to perform an inversion on the emission arising from the entire infrared, millimetre and centimetre range, making it possible to determine the dust and gas properties in many different environments in the Milky Way. The High Frequency Instrument (HFI) channels (100-857 GHz) allow us to properly constrain the big grain temperature and emissivity, while using the Low Frequency Instrument (LFI) channels $(30-70 \mathrm{GHz})$, it is possible to constrain non-thermal emission mechanisms such as free-free, synchrotron or anomalous dust. We have used these data along with other ancillary data to perform a large-scale, low-resolution analysis of the Milky Way ISM emission.

Knowledge of the dust emission and physical conditions throughout the Milky Way provides a self-consistent context for other Planck studies: for example, the environmental effect on the properties of cold cores (Planck Collaboration 2011r,s) can be evaluated, the modelling of individual anomalous dust regions can be placed in a Galaxy-wide context (Planck Collaboration 2011p), or the Milky Way values can simply be compared to other galaxies (Planck Collaboration 20111,m).

In Sect. 2 we describe the data used in the study, including both those to be inverted and those used as templates to represent a particular phase of the ISM. In Sect. 3, we describe how we optimise the separation of the gas observation into a number of Galactocentric rings, as well as how we report uncertainties on our best solution. We present the results in Sect. 4, and discuss

\footnotetext{
1 Planck (http://www.esa.int/Planck) is a project of the European Space Agency (ESA) with instruments provided by two scientific consortia funded by ESA member states (in particular the lead countries: France and Italy) with contributions from NASA (USA), and telescope reflectors provided in a collaboration between ESA and a scientific consortium led and funded by Denmark.
}

potential sources of uncertainty and bias in Sect. 5.1. Finally we conclude in Sect. 6.

\section{Data}

There are two categories of data used in this study, the data to be inverted, and data used to create templates which will be used to perform the inversion.

\subsection{Data to be inverted}

We use data from $12 \mu \mathrm{m}(25 \mathrm{THz})$ to $1.4 \mathrm{GHz}$ in order to study the dust and gas properties in a self consistent manner throughout the Milky Way. Here we briefly present these data in order of decreasing (increasing) frequency (wavelength).

The Infrared Astronomical Satellite (IRAS), a joint project of the US, UK, and the Netherlands (Neugebauer et al. 1984), performed a survey of $98 \%$ of the sky at four wavelengths: 12 , 25,60 , and $100 \mu \mathrm{m}$. We use a reprocessed version of this data set (IRIS, Miville-Deschênes \& Lagache 2005) which benefits from a better zodiacal light subtraction and from a calibration and zero level compatible with DIRBE, as well as from a better destriping.

The DIRBE instrument (Diffuse Infrared Background Experiment, Hauser et al. 1998) on board the COBE satellite imaged the full sky in 10 broad photometric bands from 1 to $240 \mu \mathrm{m}$ with a beam of $0.7^{\circ}$. The bands at 140 and $240 \mu \mathrm{m}$ allow us to bridge the gap between IRAS and the highest frequencies of HFI, thus covering the peak of the thermal dust SED.

The Planck data we used for this analysis are the DR2 LFI and HFI maps, with the cosmic microwave background (CMB) removed, as described in detail in Zacchei et al. (2011) and Planck HFI Core Team (2011b), respectively. It consists of a set of 9 frequency maps with central frequencies of 28.5, 44.1, $70.3,100,143,217,353,545$ and $857 \mathrm{GHz}$ ), smoothed to a common resolution of $1^{\circ} \mathrm{FWHM}$, at a common HEALPix (Górski et al. 2005) pixelization resolution of $N_{\text {side }}=256\left(\sim 15^{\prime}\right.$ pixels). For simplicity, in the remainder of the text we will refer to the first three bands as 30,44 and $70 \mathrm{GHz}$, respectively. The CMB component that was removed from these maps was found by the needlet internal linear combination (NILC) CMB extraction method presented in Planck HFI Core Team (2011b).

Finally, to provide constraints at radio frequencies we use the full-sky 1.4 GHz data from (Reich 1982; Reich \& Reich 1986; Reich et al. 2001).

All of the above data sets have been smoothed with a Gaussian function to a common resolution of $1^{\circ} \mathrm{FWHM}$ and gridded to a common HEALPix $N_{\text {side }}$ of 256 .

\subsection{Templates}

\subsubsection{Atomic phase}

We aim to characterise the different emission mechanisms in the different phases of the ISM (atomic, molecular and ionised). For the atomic phase we use the Leiden/Argentine/Bonn Galactic H I Survey (Kalberla et al. 2005). The survey results are expressed in antenna temperature, $T_{\mathrm{A}}$. In order to convert this into column density, the effect of optical depth should be taken into account, for example (Binney \& Merrifield 1998)

$$
N_{\mathrm{HI}}=1.82 \times 10^{-18} \times \int_{v_{1}}^{v_{2}} T_{\mathrm{s}} \times \ln \left(1-\frac{T_{\mathrm{A}}}{T_{\mathrm{s}}}\right)^{-1} \mathrm{~d} v \mathrm{~cm}^{-2},
$$


for a given spin temperature $\left(T_{\mathrm{s}}\right)$. Recent studies in the plane of the Milky Way (Dickey et al. 2003, 2009) advocate the use of a higher spin temperature of around $250 \mathrm{~K}$. This higher value is justified on the grounds that along any line of sight there is a mix of cold and warm H r gas, and so an effective spin temperature is needed to correct for the opacity. This will most certainly vary as a function of Galactic longitude. However, constraining this variation is beyond the scope of this study. We have therefore assumed a constant hydrogen spin temperature of $250 \mathrm{~K}$ for the entire Galactic plane. Varying this value in the range 150-400 K has only a small effect on our results and they remain consistant within the quoted uncertainties.

\subsubsection{Molecular phase}

We assume that the bulk of the molecular gas mass is at first order well traced by CO emission (Tielens 2005). The CO data we use come from the Composite CO Survey of Dame et al. (2001). The column density of molecular hydrogen can be expressed as a function of antenna temperature $\left(T_{\mathrm{CO}}\right)$

$N_{\mathrm{H}_{2}}=2 \times X_{\mathrm{CO}} \times \int_{v_{1}}^{v_{2}} T_{\mathrm{CO}} \mathrm{d} v$.

where $X_{\mathrm{CO}}=N\left(\mathrm{H}_{2}\right) / W_{\mathrm{CO}}$ is the ratio of molecular hydrogen column density to the velocity-integrated intensity of the ${ }^{12} \mathrm{CO}$ line. We use a value for $X_{\mathrm{CO}}$ which is compatible with the recent values from the Fermi collaboration (Abdo et al. 2010; Ackermann et al. 2011), $X_{\mathrm{CO}}=1.8 \times 10^{20}$, which results from a study on diffuse gamma-ray emission in the Galactic plane.

Both the atomic and molecular data contain line of sight velocity information. It is therefore possible to use this information to separate the emission into a set of Galactocentric rings, as a given line of sight velocity measurement uniquely identifies a Galactocentric distance, assuming circular orbits. This should not be confused with heliocentric distances for which there is a distance ambiguity corresponding to the near or far side of the derived Galactocentric orbit. The velocity information alone does not allow one to determine if the observed gas is on the near side of the determined orbit or on the far side. We separate the observed $\mathrm{H}_{\mathrm{I}}$ and $\mathrm{CO}$ observations each into a set of 5 Galactocentric rings. The reasons for choosing this decomposition is explained in Sect. 3.2

\subsubsection{Dark gas}

It has been shown (e.g., Reach et al. 1994; Grenier et al. 2005) that the $\mathrm{CO}$ and $\mathrm{H}$ i do not account for all the atomic and molecular gas in interstellar clouds: there is in addition a dark gas phase. This phase is also extensively studied in Planck Collaboration (2011o), where the total gas column density, as traced by H I and $\mathrm{CO}$ observations, is correlated with the optical depth of the dust calculated using IRAS $100 \mu \mathrm{m}$ along with Planck HFI data at 350 and $500 \mu \mathrm{m}(857$ and $545 \mathrm{GHz})$. The correlation is linear at low and high column densities, but it departs from linearity at column densities of $7.4 \times 10^{20}<N_{\mathrm{H}}<5.0 \times 10^{21}$. This excess is attributed to thermal emission by dust associated with a dark gas phase, undetected by $\mathrm{H}$ I and $\mathrm{CO}$ measurements.

The dark gas column density used here is defined in Planck Collaboration (2011o) as

$N_{\mathrm{H}}^{X}=\left(\tau_{\mathrm{D}}-\tau_{\mathrm{M}}\right) /\left(\frac{\tau_{\mathrm{D}}}{N_{\mathrm{H}}}\right)^{\mathrm{ref}}$, where $\tau_{\mathrm{D}}$ is the thermal dust optical depth, $\tau_{\mathrm{M}}=\left(\tau_{\mathrm{D}} / N_{\mathrm{H}}\right)^{\mathrm{ref}}$ $\left.\left[N_{\mathrm{HI}+2 X_{\mathrm{CO}} W_{\mathrm{CO}}}\right]\right)$ is the modelled dust opacity and $\left(\tau_{\mathrm{D}} / N_{\mathrm{H}}\right)^{\mathrm{ref}}$ is the reference dust emissivity measured in low $N_{\mathrm{H}}$ regions. We use their dark gas estimate at $857 \mathrm{GHz}$ as an additional template.

The dark gas phase represents a significant fraction of the total gas column density, as shown in Fig. 2. The H I dominates over almost the entire longitude range, and the $\mathrm{CO}$ is strongest in the molecular ring $40^{\circ}>l>-40^{\circ}$. Outside of the molecular ring the dark gas is stronger than the $\mathrm{CO}$, except towards the anti-centre direction where the dark gas and $\mathrm{CO}$ are roughly comparable. The proportion of dark gas to total hydrogen column density ranges from roughly $10 \%$ in the anti-centre direction up to nearly $60 \%$ towards the Galactic centre.

\subsubsection{Synchrotron emission}

An additional emission mechanism which is important in the frequency range we are studying and is not traced by usual gas tracers is synchrotron emission, which arises from the acceleration of cosmic-ray electrons in magnetic fields. This emission follows approximately a power law but the spectral index most certainly varies on the sky and as a function of frequency. More than $90 \%$ of the observed synchrotron emission arises from a diffuse component (Bennett et al. 2003) and in the Galactic plane recent studies have shown that there is no hardening of the synchrotron spectrum (Finkbeiner 2004; Boughn \& Pober 2007), but that emission from spinning dust is likely to dominate between 8 and $60 \mathrm{GHz}$. For our synchrotron template we have chosen the $408 \mathrm{MHz}$ all-sky survey from Haslam et al. (1982), which traces the diffuse, soft synchrotron component.

\subsubsection{Ionised phase}

To represent the ionised component we have used the maximum entropy method (MEM) free-free template from the WMAP seven year data (Jarosik et al. 2011). The intensity map $\left(I_{v}\right)$ has been converted to ionised hydrogen column density as described by Sodroski et al. (1997):

$N_{\mathrm{H}_{\text {II }}}=1.2 \times 10^{15} T_{\mathrm{e}}^{0.35} v^{0.1} n_{\mathrm{eff}}^{-1} I_{v}$

for effective electron density $n_{\text {eff }}$ and electron temperature $T_{\mathrm{e}}$. Following Sodroski et al. (1989), we use $n_{\mathrm{eff}}=10 \mathrm{~cm}^{-3}$ and $T_{\mathrm{e}}=8000 \mathrm{~K}$. In the future, the availability of radio recombination line (RRL) surveys will provide a more direct measurement of the free-free component in the Galaxy and its distribution separated into distinct Galactocentric bins using the radial velocity information included in such surveys.

As has been done for the data, the templates have all been smoothed to $1^{\circ} \mathrm{FWHM}$ and gridded to an $N_{\text {side }}$ of 256 . These templates are all displayed in Fig. 1.

\section{The Galactic inversion}

Component separation techniques have been widely tested in the framework of multi-frequency observations of the CMB, especially in the context of Planck (see Leach et al. 2008, , for a review of the methods developed within the Planck Collaboration). These methods exploit spectral and spatial correlations and independence in observations made at separate frequencies, as well as external constraints and physical modelling, in order to distinguish between different physical sources of emission. Most of them are designed to efficiently separate CMB from foregrounds and possibly foregrounds into "components": Galactic 
A\&A 536, A21 (2011)
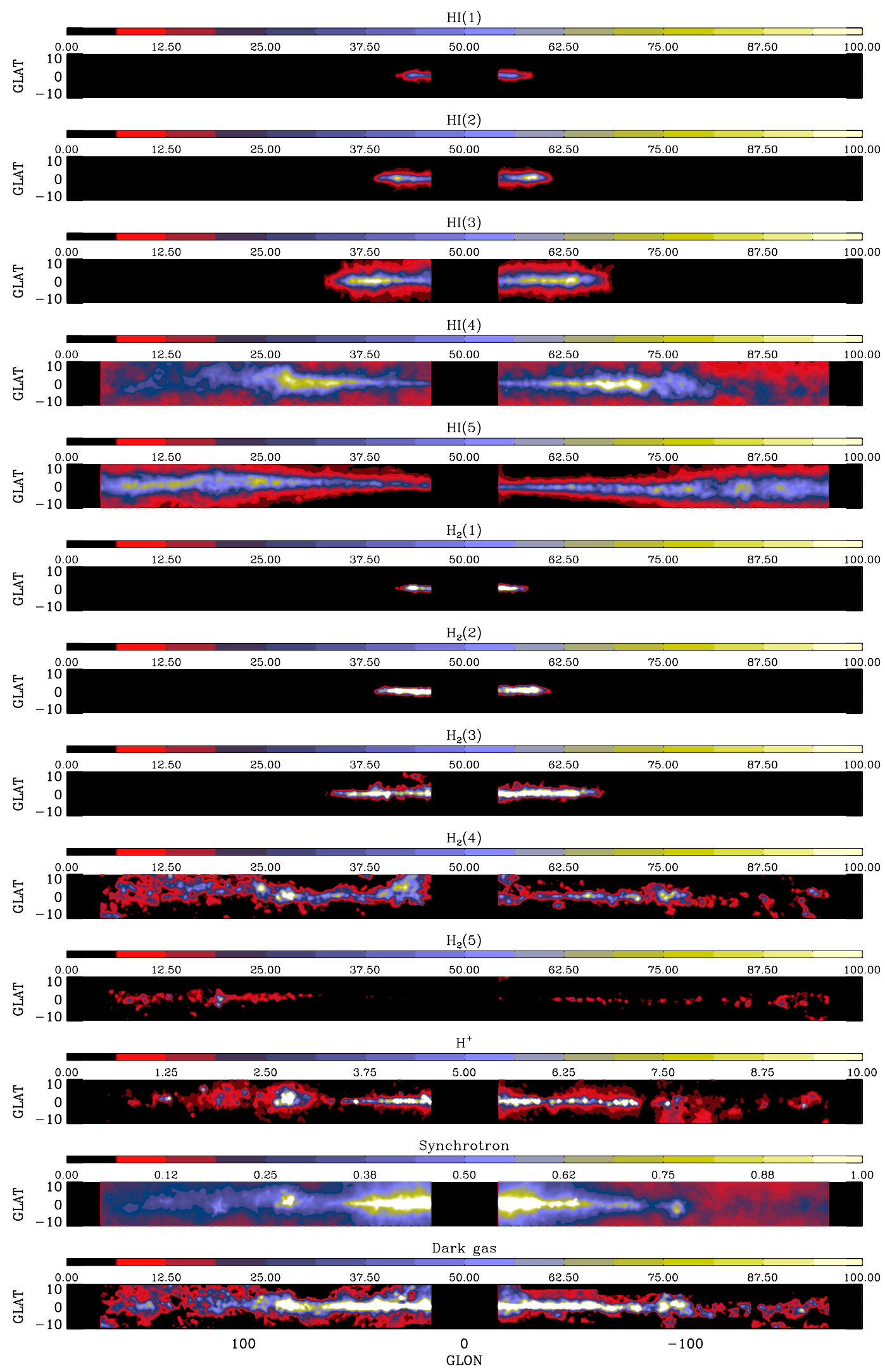

Fig. 1. Templates used in the inversion. The top five images are the $\mathrm{H}_{\mathrm{I}}$ templates, the following five are the molecular templates, all of which are presented in increasing Galactocentric radius (the radii defining each ring are described in Sect. 3.2). The final three templates are the free-free, synchrotron, and dark gas templates, respectively. The units are all expressed in $10^{20}$ atoms, except for synchrotron, expressed in $\mathrm{MJy} \mathrm{sr}^{-1}$. The centre and anti-centre regions have been masked as no distance information can be obtained from the radial velocity of the gas. 


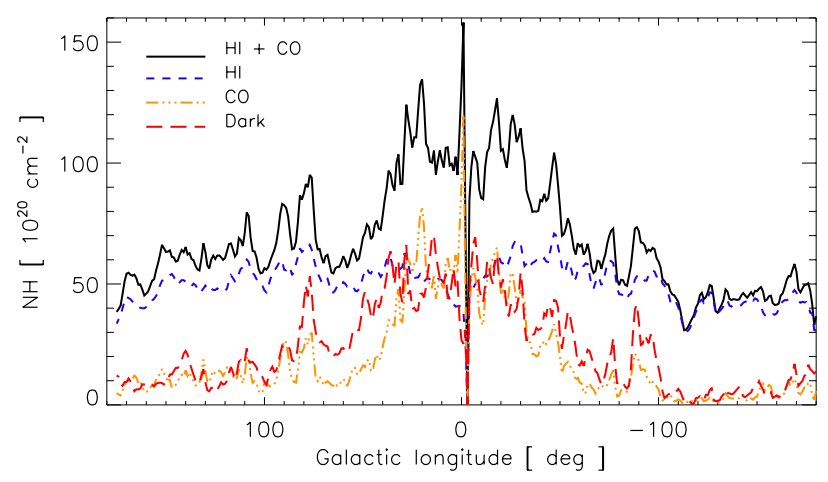

Fig. 2. Longitude profile of $\mathrm{H}_{\mathrm{I}}, \mathrm{CO}$ and dark gas. The black solid line shows the total contribution from $\mathrm{H}_{\text {I }} \& \mathrm{CO}$. The dark gas represents a significant fraction of the gas column density, and dominates the $\mathrm{CO}$ outside of the molecular ring.

synchrotron, free-free and dust emissions; extra-galactic and farIR point sources; Sunyaev-Zeldovich effect, etc.

The approach presented here is different from these methods as it tries to quantify the separate components responsible for diffuse Galactic emission. Nevertheless it is not strictly speaking a component separation method, in the sense presented above, as it fits one map of the sky with spatial templates at different Galactocentric distances and is not exploiting the spectral signature of each component to perform the separation.

\subsection{Inversion method}

We assume that each of the frequency maps $\left(I_{v}\right)$ can be expressed as a linear combination of the spatial templates described in Sect. 2.2 and presented in Fig. 1. Then, for each frequency map, we are solving for the mean emissivity of each template (in units of $\mathrm{MJy} \mathrm{sr}^{-1} / 10^{20}$ atoms) which best reproduces the observed data. Note that we are not taking into account any spatial variations of the emissivity within a given template, but are assuming a constant value for each of our templates. The modelled emission can be written, for any frequency map $I_{v}(x, y)$, as:

$$
\begin{aligned}
I_{v}^{m}(x, y)= & \sum_{i=1}^{n}\left(\epsilon_{\mathrm{H}_{\mathrm{I}}}^{i}(v) N_{\mathrm{H}_{\mathrm{I}}}^{i}(x, y)+\epsilon_{\mathrm{H}_{2}}^{i}(v) N_{\mathrm{H}_{2}}^{i}(x, y)\right) \\
& +\epsilon_{\mathrm{H}_{\mathrm{II}}}(v) N_{\mathrm{H}_{\mathrm{II}}}(x, y)+\epsilon_{\mathrm{s}}(v) N_{\mathrm{s}}(x, y) \\
& +\epsilon_{\mathrm{d}}(v) N_{\mathrm{d}}(x, y)+C_{v},
\end{aligned}
$$

where the sum is over a number $(n)$ of Galactocentric rings $(i)$ for the atomic and molecular phases, denoted $N_{\mathrm{H}_{\mathrm{I}}}$ and $N_{\mathrm{H}_{2}}$ respectively. $N_{\mathrm{H}_{\mathrm{II}}}, N_{\mathrm{s}}$ and $N_{\mathrm{d}}$ denote the ionised, synchrotron and dark gas components, respectively, and $C_{v}$ is a constant. Note that the last three templates do not have any rings, as we have no way of separating the information into different Galactocentric distances and so they are a function of frequency only. As such, the quoted $\epsilon$ are a function of $v$ only.

In order to find the values for the different emissivities, $\epsilon$, we minimise the chi-squared value defined by

$\chi^{2}=\Sigma_{j=1}^{N_{\text {pixels }}} \frac{\left(I_{\bmod }(j)_{v}-I(j)_{v}\right)^{2}}{\sigma_{v}^{2}}$,

where $\sigma_{v}$ is the noise in the map $I_{v}$, estimated as the median value of the intensity for $|b|>70^{\circ}$.

In our case, the difference between model and observations is not at all dominated by the low noise present in the Planck bands, but by the simplistic model that we are adopting for the inversion. As such we suppose that the noise does not vary spatially.

The solution which minimises Eq. (6) is found via matrix inversion. By writing the problem as

$b_{i}=a_{j} \cdot A_{i j}$,

where $b$ is the observed intensity map, $A$ is an $N \times M$ matrix of the different templates and $a$ is the vector of the parameters. The solution is simply (Press et al. 1992)

$a=\left(A^{T} A\right)^{-1} \times A^{T} b$.

The optimal mathematical solution which solves Eqs. (5)-(6) may not be physical; in particular, it may include negative emissivities. However, we do not have any cases where the emissivity is more than one sigma below zero, so remaining consistent with zero. As such we do not place any constraints on the parameters of the inversion.

\subsection{Galactocentric ring optimisation}

The observed radial velocities of $\mathrm{H}_{\mathrm{I}}$ and $\mathrm{CO}$ are used to separate the observed emission into a number of Galactocentric rings, assuming circular orbits for the gas and using the rotation curve from Clemens (1985). The choice of the distance intervals for the rings is not arbitrary, but is subject to a number of constraints. Choosing too many would create a set of rings that would be highly correlated and result in a degenerate solution. An optimised set of rings has been defined by minimising the correlation between rings, and thus minimising the degeneracy between the various components of the inversion. The total number of rings and their thickness are also driven by the requirement of a minimal number of data falling into each ring in order to constrain it and separate its contribution from the others. Further, we have chosen to fix the solar circle as one of the rings, including all velocity channels that contain high latitude, low velocity clouds.

The ring optimisation process consists first in dividing the $\mathrm{H} \mathrm{I}$ and $\mathrm{CO}$ templates into a large number of small rings, and then building the covariance matrix $\left(A^{T} A\right.$, see Eq. (8)) associated with this highly discrete basis of annuli. An iterative process is then performed: neighbouring rings with the highest correlation are merged and the new covariance matrix is updated with a reduced number of annuli. The process continues, merging the rings which have strongest correlation between them, until a reasonable number of rings is obtained. This leads to the following decomposition: $R_{\mathrm{GAL}}=[1.5,4.2,5.4,7.1,9.8,25.0] \mathrm{kpc}$, where the values define the ring edges.

\subsection{Estimating uncertainty}

The result of the inversion is an average gas emissivity at each frequency, in each gas phase and in each ring (when available). However, within a given gas phase the dust and gas properties may vary significantly and we wish to report this uncertainty. In other words, the residual differences between the model and the data are not due to noise in the data, meaning that the uncertainty on the resulting emissivities cannot be computed analytically (i.e., the residuals are not well-modelled by the uniform 

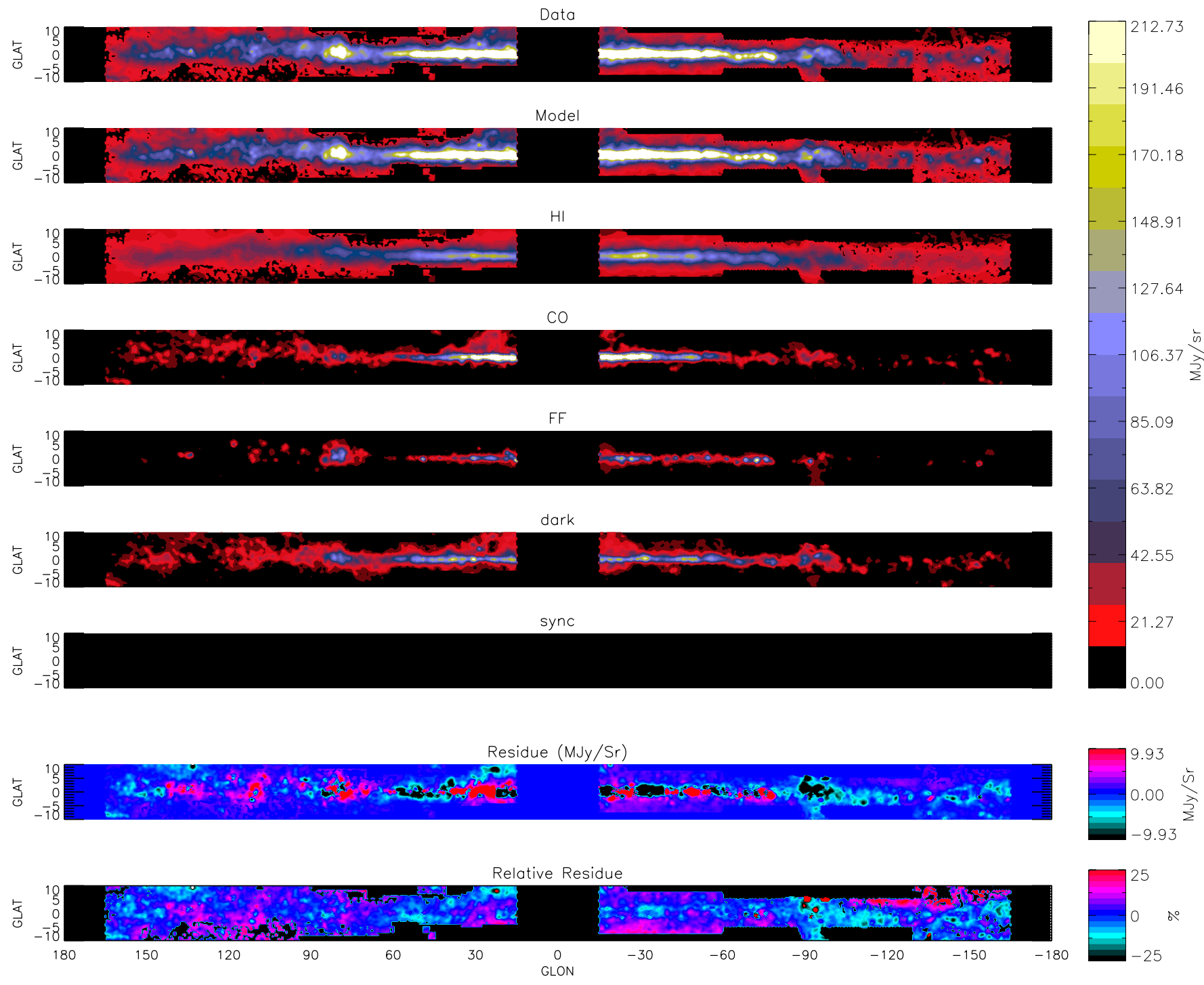

Fig. 3. Results of the inversion for the $857 \mathrm{GHz}$ band. From top to bottom are: observed emission; modelled emission; atomic contribution; molecular contribution; ionised contribution; dark gas contribution; synchrotron contribution; residual; and relative residual. All images except for the residual maps are at the same intensity scale. The centre and anti-centre regions have been masked, as the kinematic distance method is inapplicable to these regions. Areas where no $\mathrm{CO}$ observations are available have also been masked.

Gaussian noise implicit in the solution, Eq. (8)). The residuals are rather due to unknown differences between the templates and the data. An alternate method is then needed to estimate the uncertainties on the results.

The dust properties in the northern Galaxy may differ from those in the southern part, and of course the variation may be even more localised than that. To quantify this uncertainty we perform jackknife tests by masking approximately $30 \%$ of the Galaxy in 30 random blocks, each $3^{\circ}$ wide, and performing the inversion on the unmasked zones. This is repeated 500 times, and the rms value of the jackknife tests is used to provide the total variation of the emissivity within each ring and gas phase. We therefore provide robust estimates of the dust emission and provide their variation for each ring and gas phase.

It should be stressed that this estimation does not take into account the errors on either the $\mathrm{H}_{\mathrm{I}}$ opacity correction nor the uncertainty on the assumed $X_{\mathrm{CO}}$ value for each phase globally.

\section{Properties of the ISM in the Galactic plane}

The above inversion method has been applied to 16 different frequency bands from $12 \mu \mathrm{m}(25 \mathrm{THz})$ to $1.4 \mathrm{GHz}$. The results are presented in table form in the appendix (Table A.1).

Figures 3-5 compare our best-fit model to the data for three frequencies (two Planck frequencies, 857 and $30 \mathrm{GHz}$, as well as the $1.4 \mathrm{GHz}$ radio frequency) and the contributions of the different gas components to the model. The model reproduces the data rather well, especially when one notices the scale of the residual.

The main contributors to the $857 \mathrm{GHz}$ map (see Fig. 3) are the atomic and molecular phases (including the dark gas) with a modest contribution from the ionised component. At $30 \mathrm{GHz}$ (Fig. 4) the emission is a combination of the molecular, ionised, dark gas and synchrotron, with very little hydrogen. As for the solution at $1.4 \mathrm{GHz}$ (Fig. 5) it is the synchrotron component that dominates the solution, but with a significant contribution from the ionised component. 

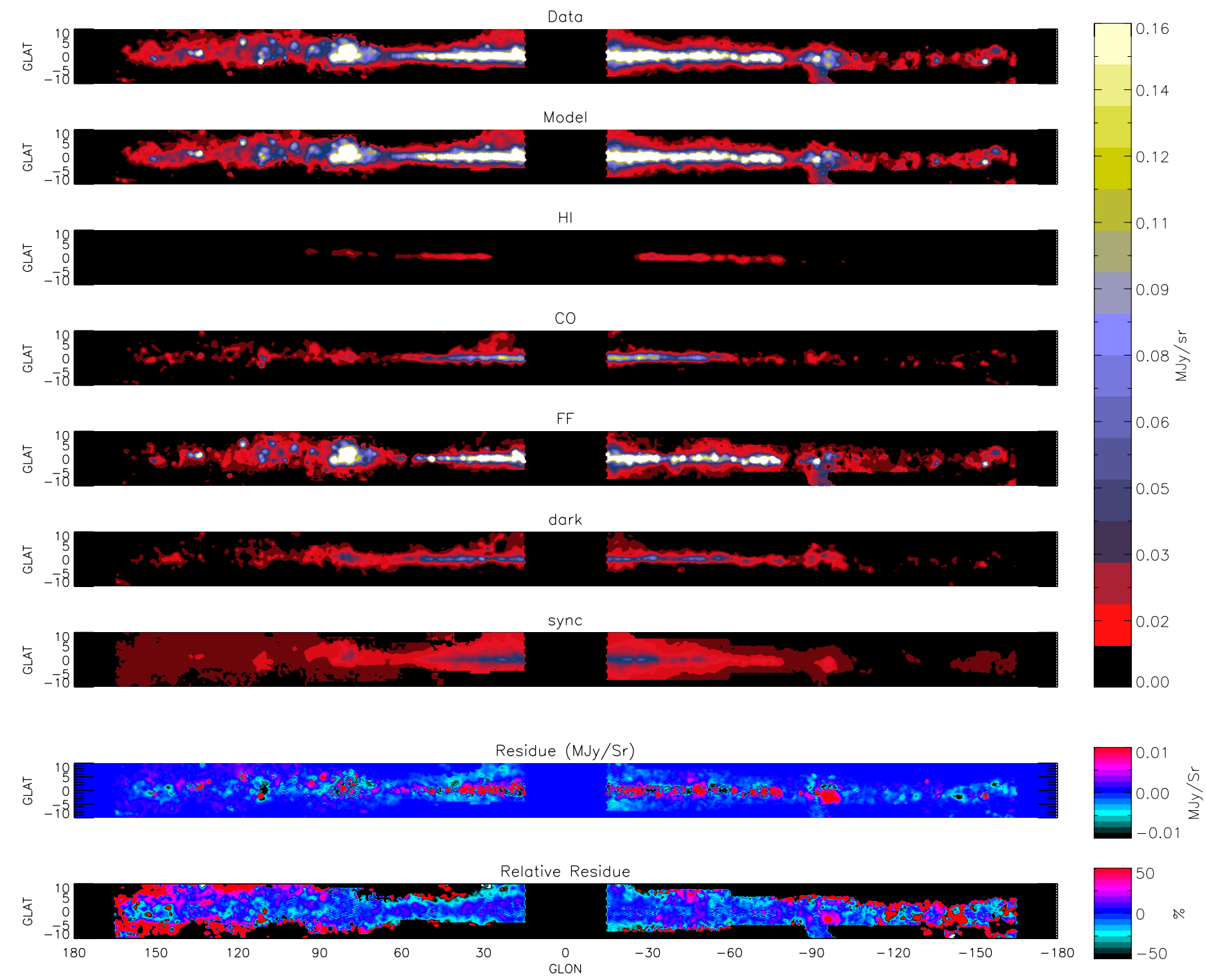

Fig. 4. Same as Fig. 3 for the $30 \mathrm{GHz}$ band.

\subsection{Dust in thermal equilibrium}

We have constructed SEDs for each of our templates across all frequencies that we have analysed. Where the emissivity is compatible with zero, we plot the $2 \sigma$ upper limit as a downward arrow.

We have fit the SEDs using a sum of thermal dust (big grains), spinning PAH molecules, free-free and synchrotron emission. The large dust grains in thermal equilibrium are well described by a modified blackbody of the form

$I_{v} \propto B_{v} \frac{v^{\beta}}{v_{0}}=\frac{2 h v^{3}}{c^{2}\left(\mathrm{e}^{h v / k T}-1\right)} \frac{v^{\beta}}{v_{0}}$,

where $T$ is the dust temperature and $\beta$ is the emissivity spectral index. When fitting using both $T$ and $\beta$ as free parameters, we find a mean value of $\beta=1.8 \pm 0.1$ throughout the Galaxy. However, to increase the reliability of the fit, we follow Planck Collaboration $(2011 \mathrm{t}, \mathrm{u}, \mathrm{o})$ and fix $\beta=1$.8. We use all data points down to $143 \mathrm{GHz}$ in the fit, but exclude $217 \mathrm{GHz}$ in the molecular and dark gas phases due to contamination from CO line emission. All points are colour corrected assuming $v I_{v}=$ constant as described in Planck HFI Core Team (2011b). The SEDs for the atomic, molecular and ionised phases are shown in Figs. 6-8, respectively. We are able to constrain the dust temperature for each component and in the various Galactocentric radii. The resulting temperature profile is shown in Fig. 9. The temperature in the atomic phase is seen to decline steadily with increasing Galactocentric distance. This decrease in temperature follows the trend of the ambient ISRF (Mathis et al. 1983), shown by the solid line in Fig. 9, indicating that the dust in the atomic phase is predominantly heated by it.

The dust in the molecular component, however, is seen to be more steady, peaking in the molecular ring ( $3 \lesssim R_{\mathrm{G}} \lesssim 6 \mathrm{kpc}$ ) and once again outside the solar circle. This phase seems to be heated by the presence of star formation. The ionised phase (dotted line) is seen to be dominated by grains that are on average much warmer than either of the other two phases. These results are in agreement with previous Galactic analyses (Sodroski et al. 1997; Paladini et al. 2007). The temperature within the solar circle $(T=17.6 \pm 0.1 \mathrm{~K})$ is completely in agreement with the average value at high Galactic latitude (Planck Collaboration 2011t).

The dust opacity at a given wavelength is defined as $\tau_{\lambda} / N_{\mathrm{H}}$ where $\tau_{\lambda}=\epsilon_{\lambda} / B(v, T)$ is the dust optical depth. The dust opacity 

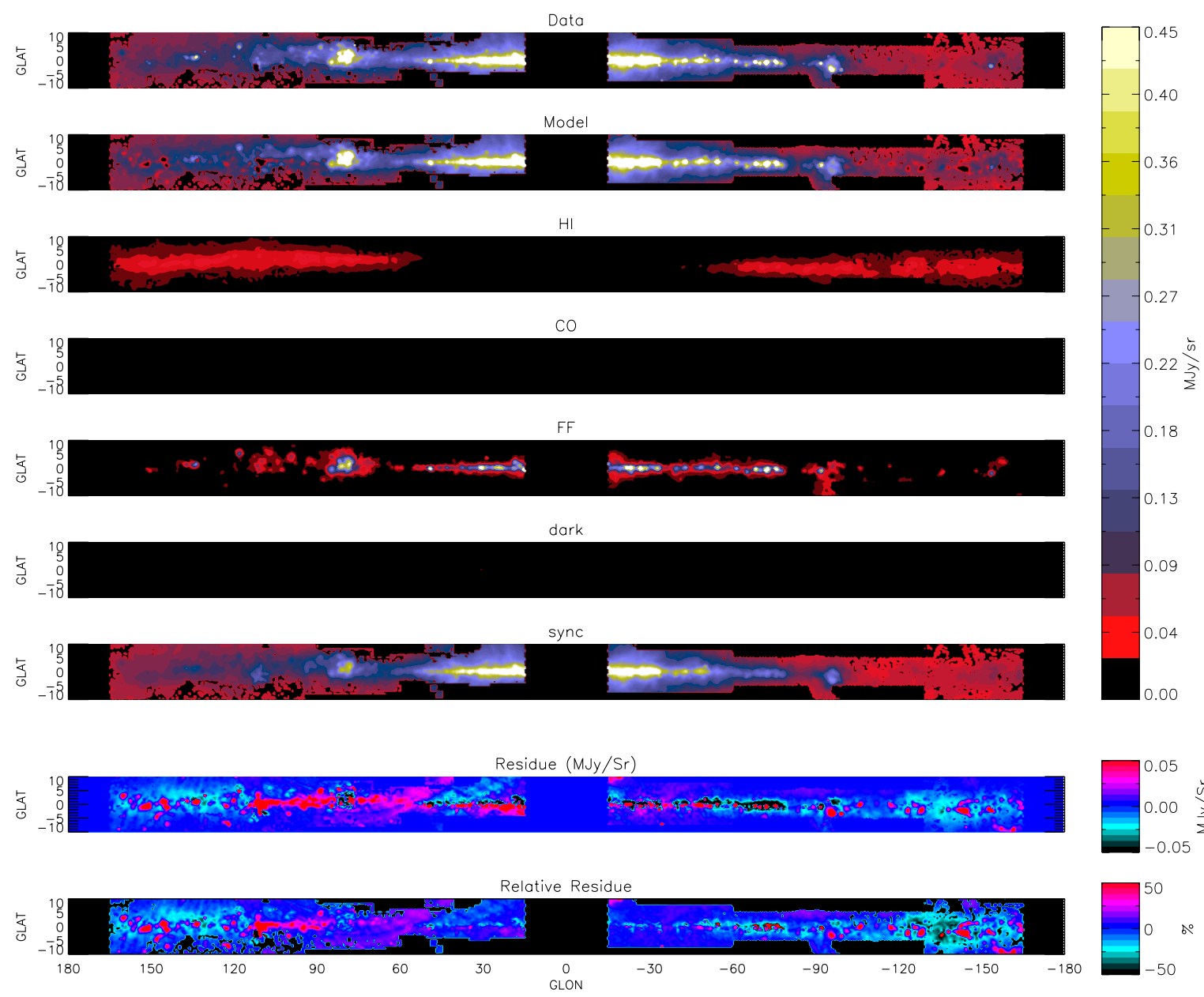

Fig. 5. Same as Fig. 3 for the $1.4 \mathrm{GHz}$ band.

in the atomic phase for the solar circle is equal to $0.92 \pm 0.05 \times$ $10^{-25} \mathrm{~cm}^{2}$ at $250 \mu \mathrm{m}$, slightly below, but in agreement with, the same value found by Planck Collaboration (2011t) at high Galactic latitude. The variation of the opacity as a function of Galactic radius is shown in Fig. 10. We do not detect any significant variation of the dust opacity as a function of Galactic radius, in either the atomic or molecular phases. Note, however, that the opacity of the molecular component is completely degenerate with the chosen value for $X_{\mathrm{CO}}$. By increasing $X_{\mathrm{CO}}$, we decrease the opacity. Further, the appropriate spin temperature for the $\mathrm{H}$ i opacity correction may differ from the constant $250 \mathrm{~K}$ we have assumed.

Finally, after fitting the thermal dust in the molecular phase, there is an obvious excess at 100 and $217 \mathrm{GHz}$ due to the presence of CO line emission in these Planck bands (Planck HFI Core Team 2011b). This is a clear indication that the inversion is working as it should, as this excess is present in the molecular phase. The excess with respect to the fit is $16.2 \pm 1.0$ and $20.2 \pm 4.5 \mu \mathrm{K}_{\mathrm{CMB}} / \mathrm{K}_{\mathrm{RJ}} \mathrm{km} \mathrm{s}^{-1}$ at 100 and $217 \mathrm{GHz}$, respectively. Planck HFI Core Team (2011b) find $14.2 \pm 1.0$ and $44.2 \pm 6.0$ at 100 and $217 \mathrm{GHz}$, respectively. At $100 \mathrm{GHz}$ our value is slightly higher, which is to be expected as it is simply an excess with respect to ${ }^{12} \mathrm{CO}$ and does not take into account the other $\mathrm{CO}$ isotopes present along the line of sight. The difference in the values at $217 \mathrm{GHz}$ is not as easy to understand, but may come from our simple assumption of a fixed $\beta$.

\subsection{Analysis of non-thermal SED components}

The SEDs for all components at frequencies lower than $100 \mathrm{GHz}$ show non-thermal dust emission features. The emission mechanisms at work in this spectral range are thermal bremsstrahlung (free-free), synchrotron emission, as well as a third commonly referred to as anomalous dust emission.

\subsubsection{Synchrotron}

The SED for the synchrotron component resulting from our inversion is shown in Fig. 11. A power-law has been recovered in the two lowest frequency LFI points and the $1.4 \mathrm{GHz}$ radio point.

For electrons with a power-law distribution of energies $N(E) \propto E^{-p}$ the frequency dependence of the emission is characterised by antenna temperature $T(v) \propto v^{\beta}$ with spectral index $\beta=-(p+3) / 2$, with typically $\beta \sim-3$. As we are not working in antenna temperature, but flux density, the power law takes the form $I_{v} \propto v^{\alpha}$ with $\alpha=\beta-2$.

We have performed a fit of the rising synchrotron spectrum with a power law and obtained a spectral index $\alpha=-1.0 \pm$ 0.1 . This value is slightly flatter than the average value found between $408 \mathrm{MHz}$ and $23 \mathrm{GHz}$ by WMAP (Dunkley et al. 2009; Davies et al. 2006). However, this value falls within the range of values found in the Galaxy (Bennett et al. 2003, $-0.5<\alpha<$ -1.1 ), and most likely represents the stronger power injection 

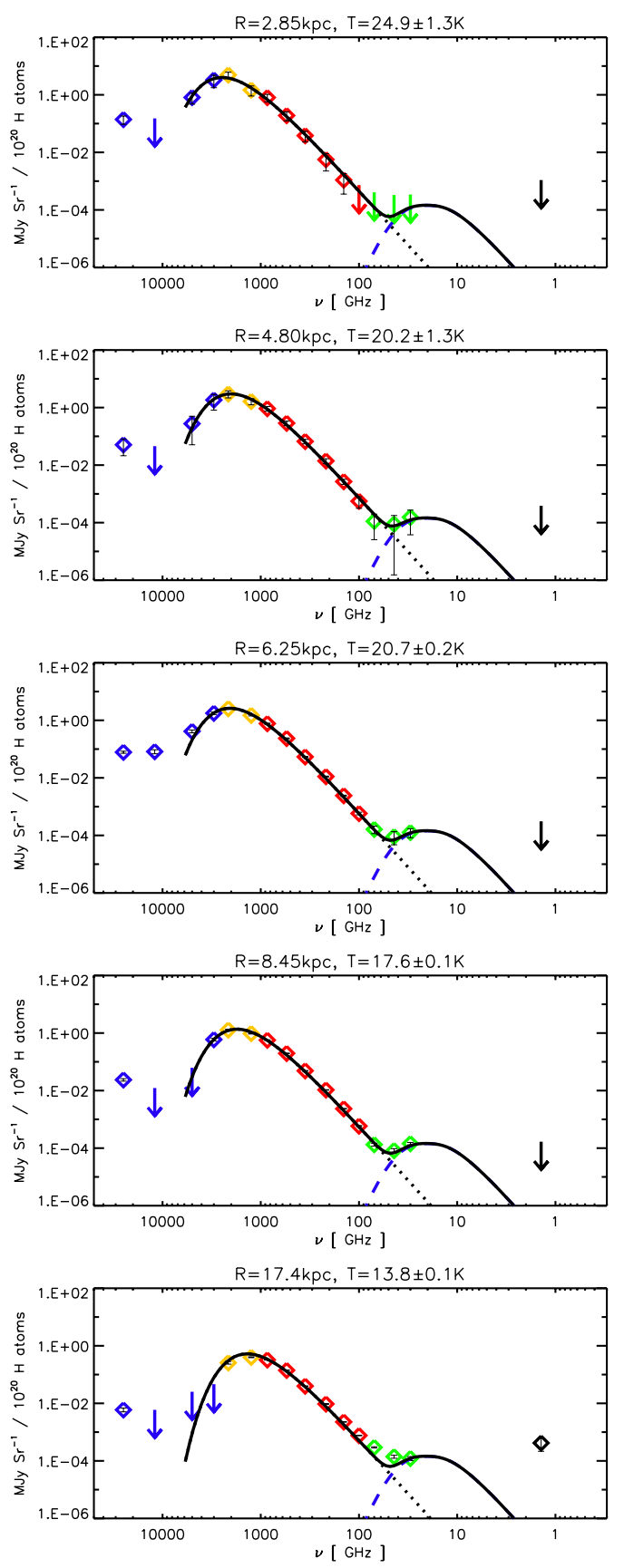

Fig. 6. SEDs for the atomic component, each sorted in increasing Galactocentric radius. The colour of the symbols refer to the mission the data came from: IRAS (blue), DIRBE (yellow), HFI (red), LFI (green) and $1.4 \mathrm{GHz}$ (black). The SEDs also show various fitted laws: the dotted line is the thermal dust SED, the short dashed line spinning dust in an atomic medium, and the solid line the sum of all contributions. The spinning dust contribution is not a fit, but simply the result of using typical values in the model (see Sect. 4.2.3 for details). The contribution of the atomic component in the outer Galaxy to the $1.4 \mathrm{GHz}$ signal is lower than the noise level derived for the $1.4 \mathrm{GHz}$ map and so should not be viewed as significant.

in the Galactic plane. There is no evidence in the data for any hardening of the synchrotron spectrum below $70 \mathrm{GHz}$.

\subsubsection{Free-free}

Free-free emission dominates the ionised hydrogen template (Fig. 8) at frequencies below $143 \mathrm{GHz}$. This emission follows
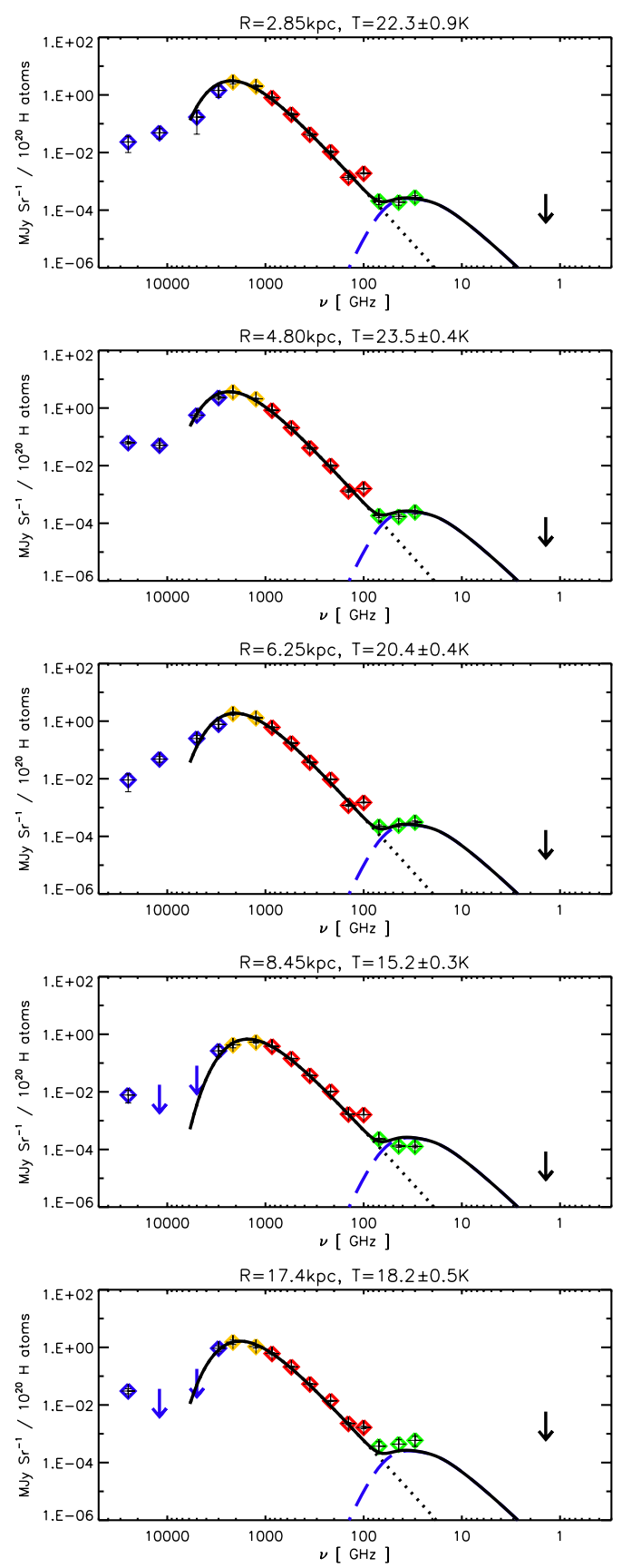

Fig. 7. SEDs for the CO component, each sorted in increasing Galactocentric radius. The different lines and colours are as described in Fig. 6, except for the long dashed line which represents spinning dust in a molecular medium.

a power-law with a spectral index of $\alpha=-0.1$ for $70 \mathrm{GHz}<$ $v<1.4 \mathrm{GHz}$. As we have used the WMAP free-free template to trace the ionised medium, it is interesting to compare the total free-free emission that we attribute to this phase with the WMAP estimate. Using the power-law fit shown in Fig. 8, we can extrapolate to the frequency of our choice and, using our $\mathrm{H}+$ template, produce an estimate of the free-free emission in any of the WMAP bands.

Performing this at $23 \mathrm{GHz}$, we recover $\sim 80 \%$ of the emission that the WMAP team find in their MEM free-free component separation. Using radio recombination lines (RRLs) from the $\mathrm{H}_{\mathrm{I}}$ Parkes zone of avoidance (ZOA) survey, Alves et al. (2010) recently showed that diffuse ionised emission can be recovered in 


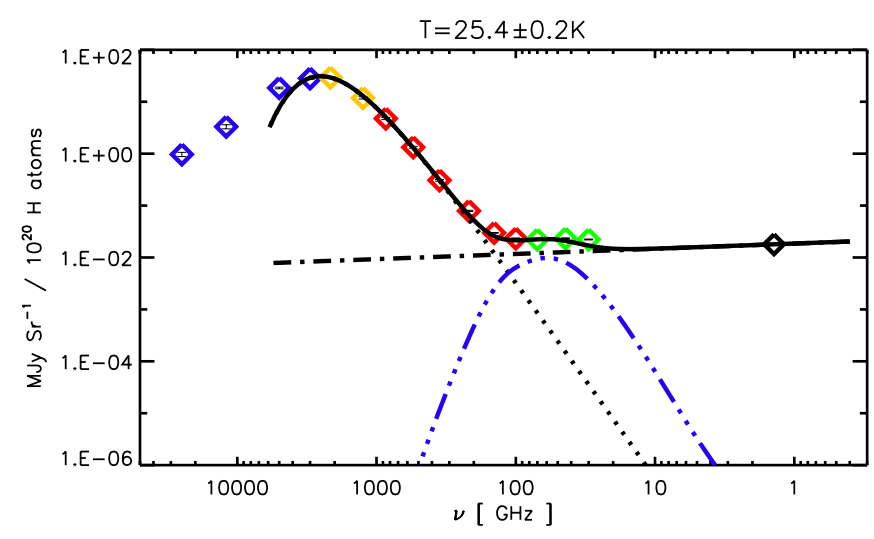

Fig. 8. The resulting SEDs for the free-free component. The different lines and colours are as described in Fig. 6, except for the dash-dot line and the dash triple dot line which represent free-free emission and spinning dust in an ionised medium, respectively.

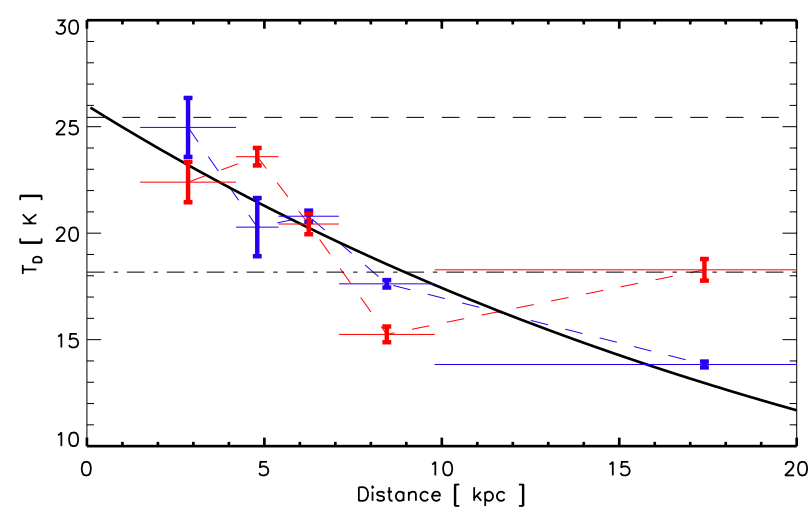

Fig. 9. Average dust temperature as a function of Galactocentric radius for the atomic (blue) and molecular (red) components. The dust temperature in the ionised phase and the dark gas phase are shown by the dashed line and dot-dashed line, respectively. The solid line shows the behaviour of the ISRF with Galactic radius. Horizontal bars show the range in distance over which the mean temperature applies. The temperature of dust mixed with atomic $\mathrm{H}_{\mathrm{I}}$ decreases with radius, consistent with the fact that this component is heated by the ambient ISRF. Dust in the molecular component is heated by embedded star formation.

the Galactic plane using RRL surveys. They then compared the level of free-free derived with the WMAP MEM estimate. As in this work, they showed it to be too high in the Galactic plane by at least $10 \%$, albeit in a much smaller $\left(8^{\circ} \times 8^{\circ}\right)$ region.

Looking at the molecular SEDs (Fig. 7), and the dark gas SED (Fig. 13), the LFI points depart from the thermal dust and seem to describe a free-free spectrum. It is not possible, however, to describe the three LFI points and the radio point using a freefree power law, except for the first and last molecular templates. Even so, all the radio points in the molecular templates are upper limits and the predicted free-free spectrum would be above the 4- $\sigma$ level for three of the templates, showing that the data are not compatible with this model. In addition, if this were indeed free-free emission, our estimate of the total Galactic free-free emission would surpass that of the WMAP MEM free-free estimate by over $30 \%$. This seems unlikely in light of the Alves et al. (2010) finding discussed above. In that case, the LFIradio points in the atomic, molecular and the dark gas phases

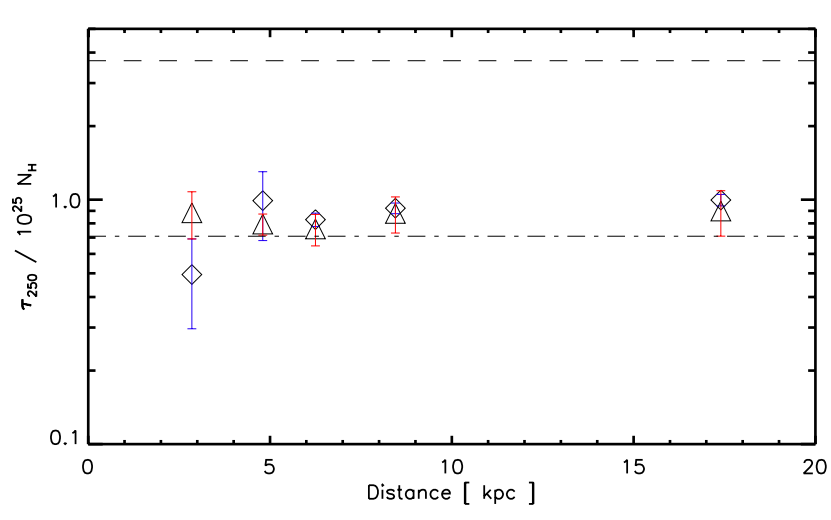

Fig. 10. Dust opacity at $250 \mu \mathrm{m}$ as a function of Galactocentric radii for $\mathrm{H}_{\mathrm{I}}$ (blue, diamonds) and CO (red, triangles). The dashed line and the dash dot line show the dust opacity for the ionised phase and the dark gas phase, respectively.

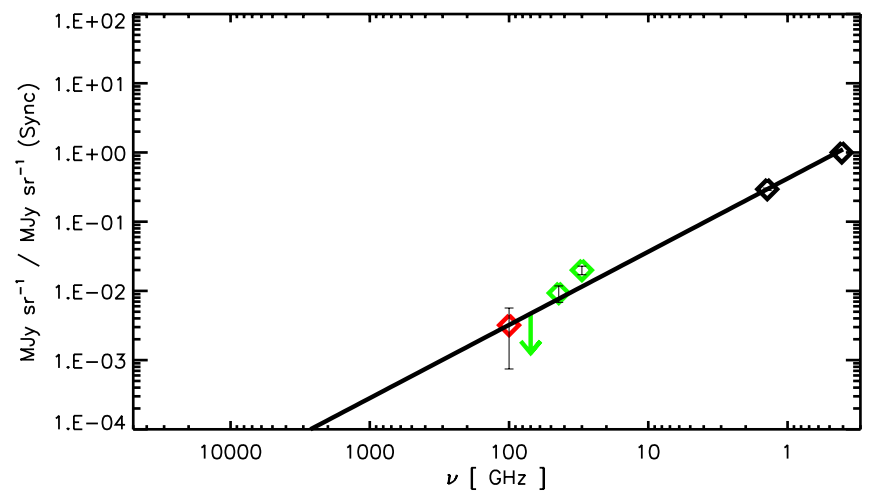

Fig. 11. The resulting SEDs for synchrotron component. The different lines and colours are as described in Fig. 6. The point at $408 \mathrm{MHz}$ is not part of the inversion, but has been plotted here to show consistency.

are anomalous microwave emission. Indeed, Boughn \& Pober (2007) recently showed that the dominant emission mechanism at $20 \mathrm{GHz}$ is spinning dust, and so is identified as anomalous microwave emission.

\subsubsection{Anomalous microwave emission}

Anomalous microwave emission, arising between 10 and $100 \mathrm{GHz}$ and first detected in the 1990s (Kogut et al. 1996), cannot be explained using classical emission mechanisms known in this frequency range (Banday et al. 2003). Indeed it is too bright to be free-free emission, and it is not polarised as thermal dust or synchrotron emissions would be. However, it is correlated with dust IR emission and especially with the interstellar PAH and very-small-grain (VSG) emission in the mid-IR (Casassus et al. 2006; Ysard et al. 2010; Scaife et al. 2010). Anomalous emission was first proposed by Draine \& Lazarian (1998) to come from rapidly rotating PAHs (Rouan et al. 1992), the spinning dust grains. Since, it has been observed in various interstellar environments: dark clouds (Watson et al. 2005; Casassus et al. 2006; Scaife et al. 2009; Dickinson et al. 2010); H II regions (Dickinson et al. 2007; Scaife et al. 2008); planetary nebulae (Casassus et al. 2007) and diffuse ISM (Miville-Deschênes et al. 2008). Several processes can excite or damp the grains' rotation: pho- 

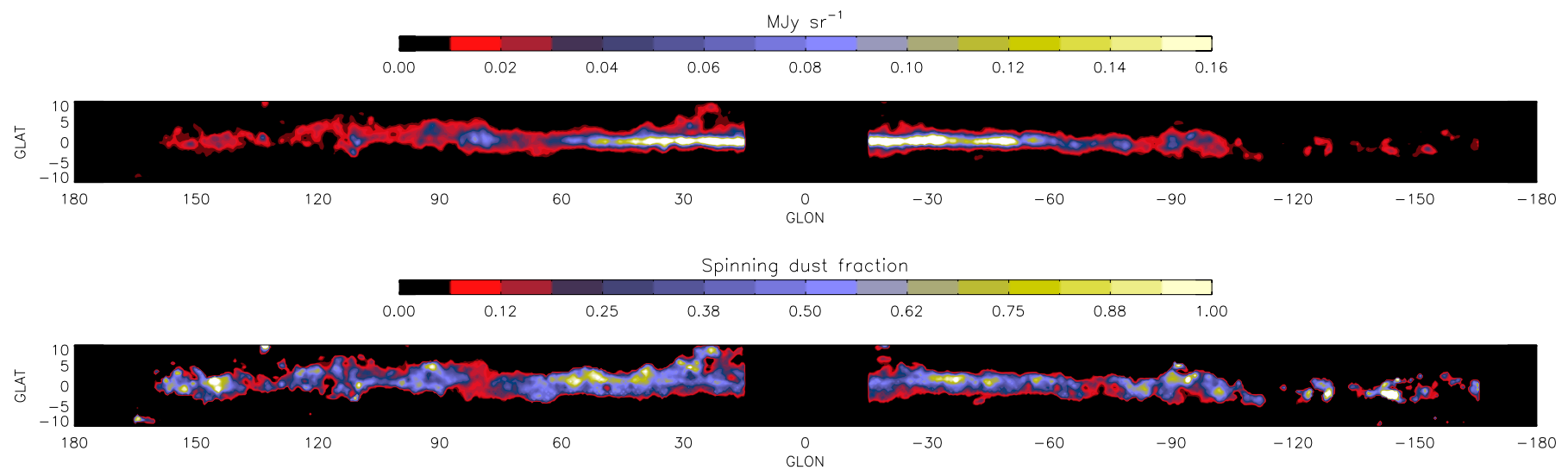

Fig. 12. Depiction of the spinning dust emission at $30 \mathrm{GHz}$ in the Galactic plane in $\mathrm{MJy} \mathrm{sr}^{-1}$ (top) and as a fraction of the total $30 \mathrm{GHz}$ signal (bottom). In making this image, it is assumed that the $30 \mathrm{GHz}$ emission associated with the atomic, molecular and dark gas phases are solely due to spinning dust. Some features may be simply due to residuals in our inversion; however, this illustrative view highlights regions where spinning dust may be particularly strong compared to other emission mechanisms.

ton emission (IR and radio); gas/grain interactions; formation of $\mathrm{H}_{2}$ molecules on the grain surface; or photoelectric emission.

Here we investigate the anomalous component in COcorrelated emission. All the rings exhibit a flattening of the SED in the three LFI bands (Fig. 7), as well as upper limits on the emission at $1.4 \mathrm{GHz}$. This clear excess in the microwave range cannot be free-free emission due to the reasons discussed in Sect. 4.2.2. The peak frequency of this excess lies approximately from 20 to $60 \mathrm{GHz}$.

We attempt to fit the excess with the spinning dust model described in Silsbee et al. (2011). Models show that spinning dust emission is sensitive to the grain size distribution, to the intensity of the ISRF, and to the density of the medium in which the grains are embedded (Ali-Haïmoud et al. 2009; Ysard \& Verstraete 2010). The smallest grains, namely the PAHs, are responsible for most of the spinning dust emissivity. Fitting the thermal dust emission with a modified blackbody, we estimate the radiation field to be close to the standard ISRF (Mathis et al. 1983; Porter et al. 2008). To describe the small dust grains, we use two grain populations: PAHs and VSGs. We adopt a size distribution that is the sum of two log-normal components (Weingartner \& Draine 2001). The first one is peaked at $3 \mathrm{~nm}$ (VSG), and the second one at $0.6 \mathrm{~nm}$ (PAHs), in line with Compiègne et al. (2011), with the width of both log-normal components set to 0.4 . Most of the spinning dust emission comes from the smallest grains, the second log-normal component, for which we assume an abundance equal to the value measured in the solar neighbourhood. Finally we describe the grains' environment as molecular gas with typical parameters $\left(n_{\mathrm{H}}=350 \mathrm{~cm}^{-3}\right.$ and $\left.T_{\text {gas }}=20 \mathrm{~K}\right)$.

The fit to the data with this simple model is surprisingly good, and shows that not only is anomalous emission present in the molecular phase throughout the Milky Way, but that spinning dust provides a compelling model with which to describe it. The predominance of spinning dust in the molecular phase is in agreement with more detailed modelling of individual anomalous emission regions (Planck Collaboration 2011p).

The ISRF could in theory also have an effect on this emission mechanism, and the big grain temperature gives us an idea of its fluctuations: $\chi=(T / 17.5 \mathrm{~K})^{\beta+4}$, where $\chi$ is dimensionless and equal to one in the solar neighbourhood. However this range of ISRF fluctuations has little effect on the spinning dust spectrum (Ali-Haïmoud et al. 2009; Ysard \& Verstraete 2010), so we as- sume a solar value for all SEDs. The average density of the molecular gas may vary with radius, which would have enough impact to significantly alter the fit. However, we have not proceeded with a detailed fit of this component, as there are many parameters and the solution would be degenerate (e.g. grain size distribution, gas density, ISRF intensity, as well as radiative transfer considerations). We have kept the analysis simple, which allows us to say that we have definitely detected anomalous microwave emission, that it is associated with the molecular phase, and that spinning dust provides a good model with which to understand the spectrum of this excess emission. Some of this emission could be explained by free-free emission, but it cannot be the dominant emission mechanism in this phase.

To study the possibility that spinning dust emission is present in either the atomic or ionised phase, we have also estimated its contribution to these. The different environments will have an impact on the spinning dust emission, so we have adapted the gas parameters in the modelling. For the atomic phase we have used the results of Heiles \& Troland (2003) and modelled the atomic ISM as composed of $40 \%$ cold neutral medium (CNM) and $60 \%$ warm neutral medium (WNM). The parameters for these phases are $n_{\mathrm{H}}=30 \mathrm{~cm}^{-3}$ and $T_{\text {gas }}=100 \mathrm{~K}$ for the CNM, and $n_{\mathrm{H}}=$ $0.4 \mathrm{~cm}^{-3}$ and $T_{\text {gas }}=6000 \mathrm{~K}$ for the WNM. As can be seen in Fig. 6, the SEDs show that spinning dust emission is able to reproduce the departure from the thermal dust spectrum for $v<$ $70 \mathrm{GHz}$. The $1.4 \mathrm{GHz}$ point in the last atomic template is almost certainly an artifact. The atomic and synchrotron templates both describe diffuse smooth emission in the Galaxy, leading to some "crosstalk" between the two components. However it should be noted that when the emissivity found for the atomic phase in the outer Galaxy is multiplied by the column density of hydrogen in the template, the total emission is very low, lower than the noise level derived for the $1.4 \mathrm{GHz}$ map. As such, it should not be viewed as significant.

For the ionised phase, we use the same gas parameters as in Sect. 2.2, namely $n_{\mathrm{H}}=10 \mathrm{~cm}^{-3}$ and $T_{\text {gas }}=8000 \mathrm{~K}$. The SED in Fig. 8 shows that spinning dust may also be present in this phase, but that it is completely dominated by free-free emission. In fact, the situation may be even worse, as we have assumed solar value PAH abundance, whereas recently Dobler et al. (2009) showed that the PAH abundance in this type of region is about a factor of three lower. 


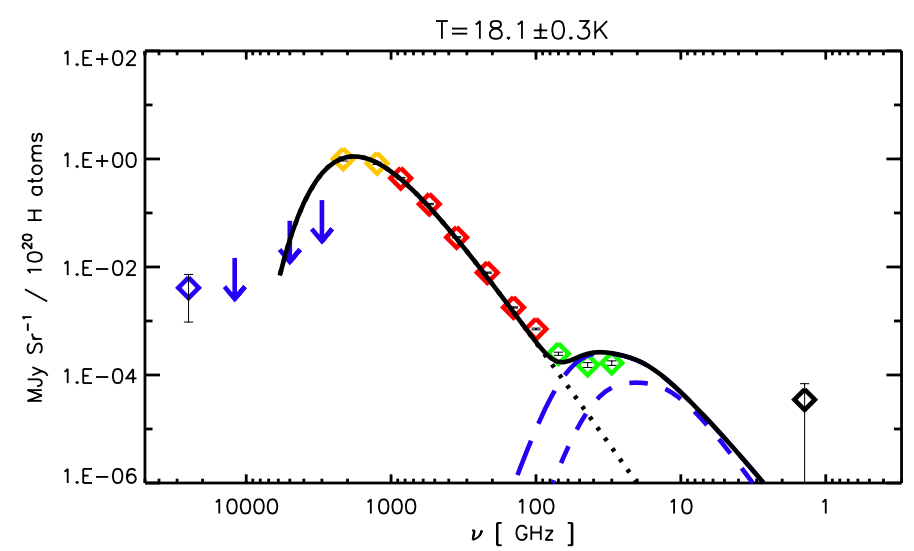

Fig. 13. The resulting SED for the dark gas component. The different lines and colours are as described in Fig. 6. The dashed and shortdashed lines shows the spinning dust contribution from a molecular phase, and from an atomic phase, respectively.

Assuming that the atomic, molecular and dark gas contributions at $30 \mathrm{GHz}$ are due solely to spinning dust, and that the ionised phase has a negligible contribution, we have produced a Galactic-plane image of spinning dust emission. This provides a complementary image to that presented in Planck Collaboration (2011p), as it has been obtained using a different approach. The intensity map is shown at the top of Fig. 12; at the bottom we show the fraction of the total signal at $30 \mathrm{GHz}$ due to spinning dust. Several bright regions show up in this map, highlighting areas where spinning dust emission is the dominant source of emission at these frequencies. Overall, spinning dust accounts for $25 \pm 5 \%$ (statistical) of the total emission at $30 \mathrm{GHz}$. This error estimate does not take into account any systematic uncertainty due to our simple model, which is difficult to quantify, and so will likely be higher.

\subsection{Dark gas}

Dark gas has been detected in numerous studies (Reach et al. 1994; Grenier et al. 2005) as an infrared, and a correlated infrared/gamma-ray, excess over and above what is expected given the gas column density derived from $\mathrm{H}$ I and $\mathrm{CO}$ data. The inversion performed without this component results in positive and negative residuals, as the other components compete to account for this missing gas phase. Not only does adding this component lower our reduced $\chi^{2}$ fits across all bands, but it also allows us to examine the nature of this dark component.

Looking at Fig. 13, the resulting SED for this component looks quite similar to both the atomic and molecular SEDs. Its temperature does not differentiate it immediately from either of the two phases, and it shows a low-frequency tail beyond the thermal dust SED. These points look similar to the anomalous dust in the molecular phase (dashed line); however, the spinning dust model lies above the LFI points. Assuming that the spinning dust is in an atomic phase (dotted line), it can be seen that it does not predict the observed emission. It thus seems to be more of a mix of the two phases, with a larger influence from the molecular phase. The different parameters discussed in Sect. 4.2.3 could also be altered, but we do not have enough data to constrain such a fit.
The interpretation of these results is somewhat complicated for several reasons. Firstly, the amount of dark gas is not expected to be independent of Galactic position, but using a 2D template we are forced to assume just that. Secondly, the estimate of this gas phase was obtained at high galactic latitude and we are using an extrapolation into the Galactic plane. It would be preferable to use an independent template, derived from gamma-ray observations for example, in future inversion analyses. Reconstructing the line-of-sight distribution of the dark gas would enable a more exact determination of the properties of the dark gas, by coupling the analysis with a 3D extinction map (e.g. Drimmel et al. 2003; Marshall et al. 2006; Sale et al. 2009). This type of analysis will be considered in a future study of the Galactic inversion.

In summary, the dark gas SED is consistent with molecular gas, most likely diffuse $\mathrm{H}_{2}$ and optically thick $\mathrm{CO}$. The best fit at longer wavelengths comes from the spinning dust model, in a molecular medium.

\section{Possible sources of bias}

Our inversion algorithm determines the flux density per column density of each template, and hence the result depends on the different templates themselves. Care should therefore be taken to use the most physically representative input for each phase of the ISM that we want to include in the inversion. Nonetheless, the jackknife tests described in Sect. 3.3 show that the method is self-consistent. Moreover, many different combinations for the $\mathrm{H}$ I+CO Galactocentric rings has been attempted, and the main results presented here remain coherent with those. However, the results are notably sensitive to one particular input, the synchrotron template. Without it, the $\mathrm{H}_{\mathrm{I}}$ templates trace the Galactic synchrotron emission, as the $\mathrm{H}_{\mathrm{I}}$ observations trace the largescale smooth structure of the Milky Way, similar to the diffuse soft synchrotron emission. This artificial component in the $\mathrm{H}_{\mathrm{I}}$ templates then masks any contribution for the anomalous microwave emission. We have attempted to trace the synchrotron emission with two different synchrotron templates: the WMAP MCMC synchrotron template (Gold et al. 2009), and the $408 \mathrm{MHz}$ full sky survey (Haslam et al. 1982). We have decided to use the latter as it more successfully removes the synchrotron contribution from the $\mathrm{H}$ i templates and allows us to detect anomalous emission in the atomic phase.

\subsection{Correlation between parameters and uniqueness of the solution}

In Fig. 14 we show the correlation between the different emissivities of our best solution at $30 \mathrm{GHz}$, where many emission mechanisms are present and the inversion is more challenging. These were obtained from the result of the jackknife tests presented in Sect. 3.3. It can be seen that for almost all the templates the correlation is low. Three elements are to be highlighted. The first one is that the emissivities for the outer Galaxy molecular template, as well as the ionised template, both have pronounced departures from a Gaussian distribution. Looking at Fig. 1, both templates are patchy; the masking of several regions simultaneously will change the morphology of each map significantly and therefore have a large impact on the derived emissivity.

Secondly, rings $1-3$ of the atomic and molecular phase are slightly anti-correlated (the contours are elliptical). This region of the Galaxy is indeed difficult to disentangle, and due to the low filling factor of these rings the algorithm has less to work 


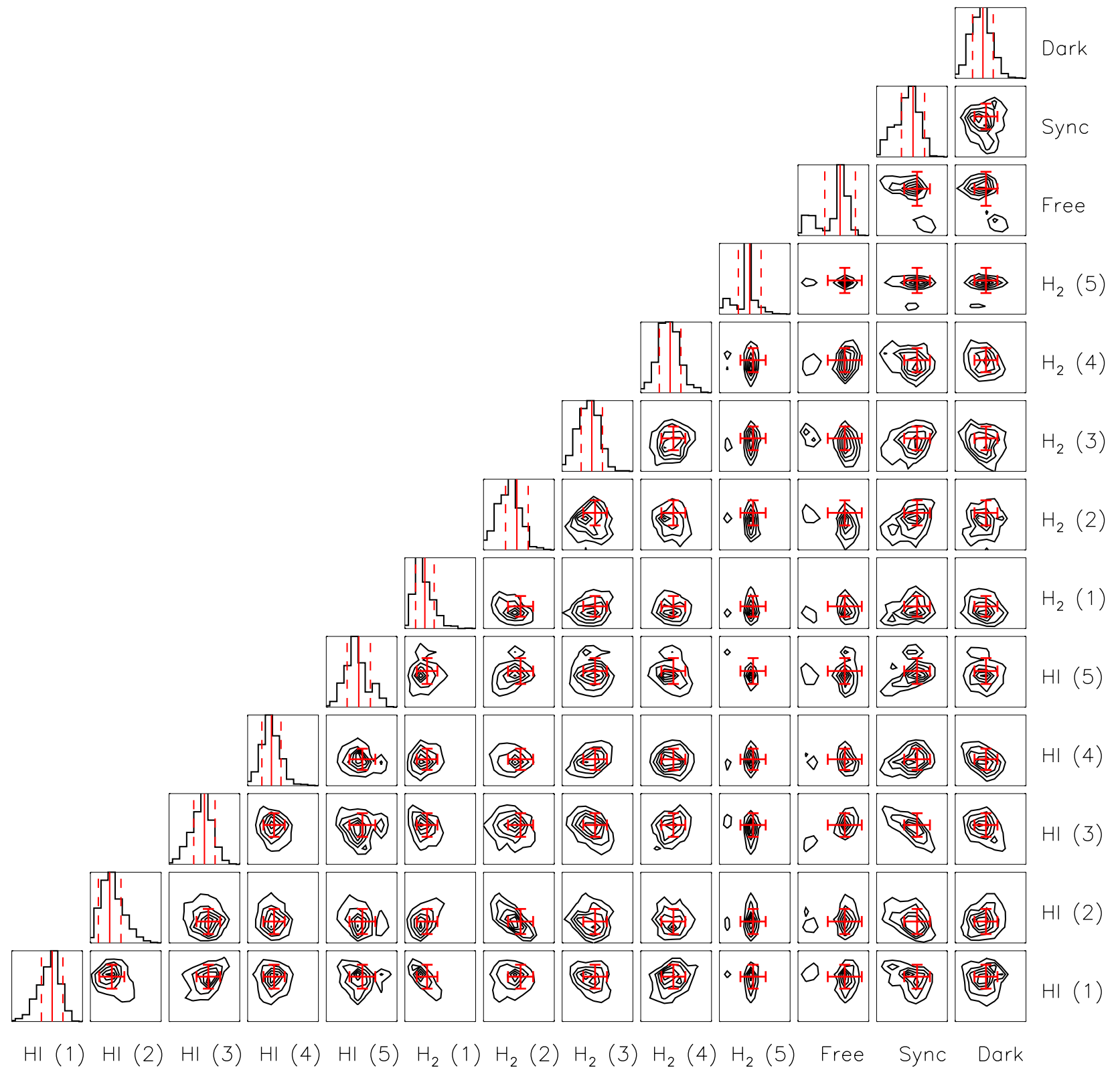

Fig. 14. Correlation matrix of the emissivities for each ring at $30 \mathrm{GHz}$. Overplotted in red are the results with corresponding error bars. Onedimensional histograms replace the contour plots where only one component is concerned.

with than in the solar circle, for example. Nevertheless the error bars deduced from the jackknife analysis account for this slight degeneracy. Higher resolution maps will help reduce this crosstalk between templates in future analyses.

Thirdly a small bias can be seen, as the results of the inversion are not always centred in the middle of the jackknife distributions. We calculated this bias by looking at the difference between the mean deviation of each result with respect to the jackknife realisations, in units of result uncertainty. That is the bias $B$ per template $i$ and per band $v$ can be written:

$B(i, v)=\frac{1}{\sigma(i, v)} \frac{1}{N_{\mathrm{r}}} \Sigma_{r=1}^{N_{\mathrm{r}}}\left[\epsilon(i, v)-\epsilon_{J}(r, i, v)\right]$,

where $N_{\mathrm{r}}$ is the number of jackknife realisations, $\epsilon(i, v)$ and $\sigma(i, v)$ are the result and uncertainty of the inversion, and $-\epsilon_{J}(r, i, v)$ is the result of one jackknife realisation. We have found this effect to be small, with a median bias of $0.005 \sigma$ over all templates and frequencies, and with a mean absolute deviation of $0.08 \sigma$. In all cases, the bias is always lower than $0.25 \sigma$.

\subsection{Effect of $C M B$ subtraction}

The CMB removal in the Planck maps we have used may create significant residuals in the Galactic plane. Therefore, to test the impact of the CMB removal on our results, a different $\mathrm{CMB}$ estimate was reconstructed through a classical internal linear combination (ILC) by means of Lagrange multipliers (Eriksen et al. 2004). $A \pm 15^{\circ}$ strip in Galactic latitude was extracted from the HFI CMB frequency channels maps (100, 143, 217 and $353 \mathrm{GHz}$ ) reduced to a common resolution (10 arcmin), in units of $K_{\mathrm{CMB}}$. This $\mathrm{CMB}$ component was subtracted from all 


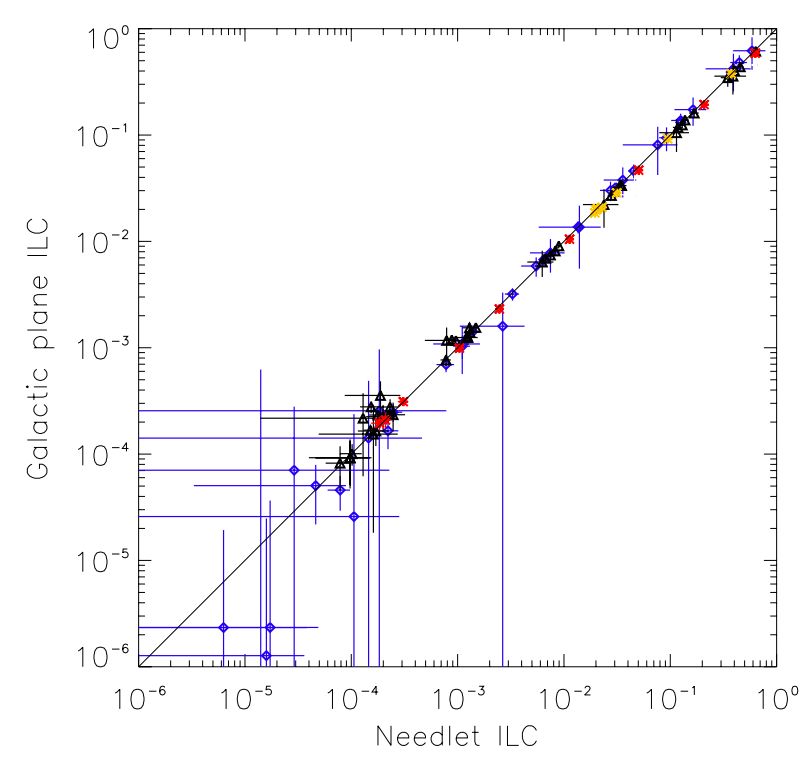

Fig. 15. Comparison of emissivities derived after application of two different CMB subtractions, the official Needlet ILC and the Galactic plane ILC. The atomic templates are the blue points, molecular are black, ionised is orange, dark gas is red and synchrotron is green.

the Planck LFI and HFI channels maps, resulting in a new and independent set of CMB-removed Planck maps.

Even though the methods are similar, the resulting CMB in the Galactic plane is quite different. In constructing the the standard CMB subtraction (Needlet ILC, "NILC"), an intensity mask was applied (Planck HFI Core Team 2011b) which results in CMB residuals remaining in a substantial part of the Galactic plane. No such mask was used in the Galactic plane ILC. Outside of this mask, the differences between the two CMB estimates are of the same order as the residuals of our fit at low frequencies, $44 \mathrm{GHz}<v<143 \mathrm{GHz}$.

Using these maps to perform the same inversion, we have concluded that the impact is small, and almost always within the error bars. In Fig. 15 we plot the derived emissivities using the NILC and the Galactic plane centred CMB removal ("ILC").

To get a better idea of where the solution might be off by more than $1 \sigma$, we show the difference in units of $\sigma$ per band in Fig. 16. The value plotted is, for each component:

$\Delta_{\sigma}=\frac{\epsilon_{\mathrm{ILC}}-\epsilon_{\mathrm{NILC}}}{\left.\sqrt{(} \delta \epsilon_{\mathrm{ILC}}^{2}+\delta \epsilon_{\mathrm{NILC}}^{2}\right)}$.

The agreement is generally very good, except for some points concentrated in the bands 143,100 and $70 \mathrm{GHz}$. In these cases the $\mathrm{CO}$ templates are higher in the Galactic plane ILC whereas the $\mathrm{H} \mathrm{I}$ and $\mathrm{H}^{+}$templates are lower. Nevertheless, there are no points that are different by more than $3 \sigma$. Therefore, these differences do not change our main conclusions, but would slightly modify the amount of anomalous emission, for example. As we are not modelling the specifics of any emission mechanisms at the lower frequencies, we are not sensitive to the impact of the CMB removal for this study.

\subsection{Impact of using dark gas template}

The dark gas template used in this study has been calculated by Planck Collaboration (2011o). It is the result of finding at what column densities the correlation between total hydrogen column density $\left(\mathrm{H} \mathrm{I}+2 \mathrm{H}_{2}\right)$ versus optical depth of the dust departs from linearity. The frequencies used to obtain the map at $857 \mathrm{GHz}$ that we use as a template are 857 and $545 \mathrm{GHz}$, along with the IRAS $100 \mu \mathrm{m}$ band. There is some circularity in using a template based on a residual in the Planck data to fit the same data. However, the data used is restricted to the two highest HFI frequencies. Here we present the impact at $30 \mathrm{GHz}$, which in no respect suffers from the same circularity, as the data are far from the frequencies used to derive the dark gas template, and they come from LFI, a completely separate instrument from HFI.

We have performed the inversion with and without the dark gas template at $30 \mathrm{GHz}$, and looked at the correlation between the residuals and the column density of the dark gas. The results are shown in Fig. 17; the inversion performed without the dark gas template is on the left, the same inversion with the dark gas template is on the right. A distinct component of the residuals can be seen to correlate very well with the dark gas column density when the dark gas template is not used. This correlation completely disappears when it is used. In addition, the reduced $\chi^{2}$ for the fit shows a significant improvement of approximately $50 \%$.

The choice of both the opacity correction for the $\mathrm{H}_{\mathrm{I}}$ and the $X_{\mathrm{CO}}$ value chosen will have an impact on our results. We have chosen to keep the analysis simple for this study, as the spin temperature we assume, together with the dark gas template, should account for most of the optically thick gas in the plane. The ability of the dark gas template used to adequately account for all the optically thick gas remains to be studied, so future work could involve using more sophisticated methods to account for this effect.

\section{Conclusions}

The analysis performed in this study provides a realistic description of the dust and gas properties as a function of Galactic radius in the Milky Way. The dust temperature in the $\mathrm{H}_{\mathrm{I}}$ is seen to decrease as a function of Galactocentric distance from 24.0 to $13.9 \mathrm{~K}$, and the temperature in the molecular phase is heated by star formation associated to the Galactic spiral arms. The opacity found for grains in the solar circle is $\tau / N_{\mathrm{H}}=$ $0.92 \pm 0.05 \times 10^{-25} \mathrm{~cm}^{2}$ at $250 \mu \mathrm{m}$, and with no significant variation with Galactic radius, even though the dust temperature is seen to drop by over $10 \mathrm{~K}$ from the centre to the outer Galaxy. The dust temperature in the $\mathrm{H}_{\mathrm{I}}$ gas in the solar circle, $17.6 \pm 0.1 \mathrm{~K}$ is also compatible with the recent Planck value of high-latitude cirrus $(17.8 \pm 0.9 \mathrm{~K})$.

The extension of our analysis to lower frequencies than previously used in inversion techniques has allowed us to place constraints on the free-free, synchrotron and anomalous microwave emission:

- anomalous dust emission is clearly seen in the atomic, molecular and dark gas phases. It is well fit by a very simple model consisting of spinning PAH molecules embedded in each of the gas phases. We highlight regions where spinning dust emission may be strong with respect to other emission mechanisms. According to our simple model, in the Galactic plane spinning dust accounts for $25 \pm 5 \%$ (statistical) of the total emission at $30 \mathrm{GHz}$. However, systematic uncertainties linked to our model may make this value more uncertain;

- the dark gas phase has been explored spectrally across the Galactic plane for the first time. Its SED is similar to the molecular phase and therefore this phase seems to be tracing 

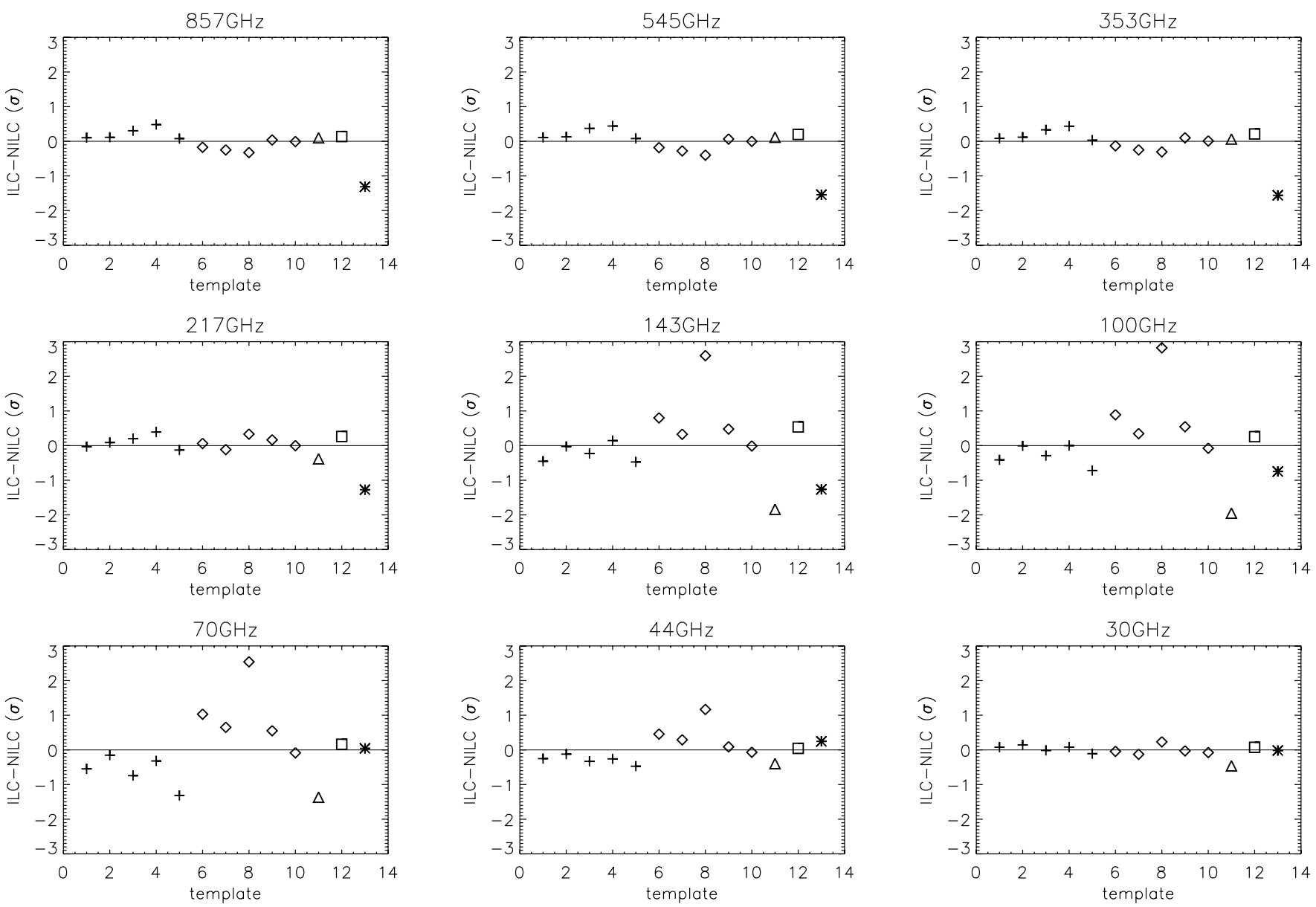

Fig. 16. Comparison of emissivities derived after application of two different CMB subtractions. The value plotted is shown in Eq. (11). The symbols represent $\mathrm{H}_{\mathrm{I}}$ (plus), $\mathrm{H}_{2}$ (diamond), $\mathrm{H}^{+}$(triangle), Synchrotron (square) and dark gas (asterisk). Differences are generally small, but become larger in the range $143-44 \mathrm{GHz}$.
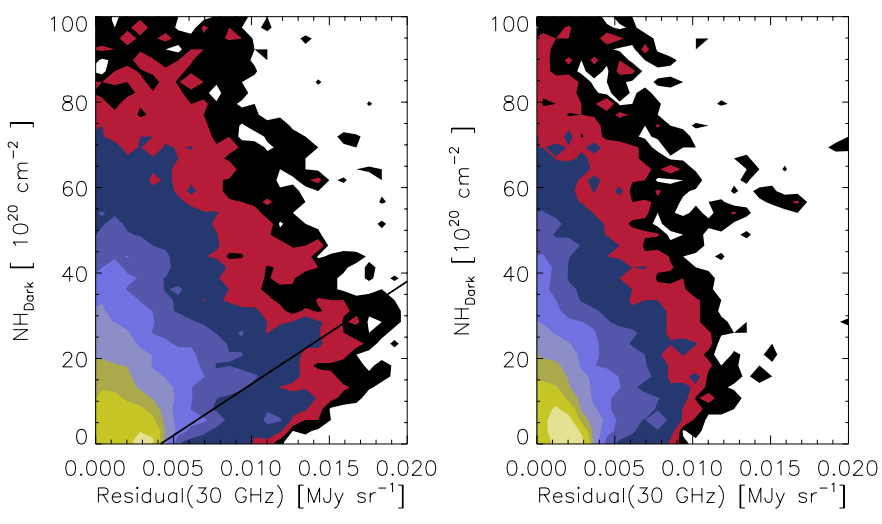

Fig. 17. Dark gas intensity as a function of the residuals of the inversion at $30 \mathrm{GHz}$ without (Left) and with (Right) the dark gas template. A distinct component of the residual (shown with the solid line) is seen to be correlated with the dark gas template. This correlated residual disappears when the dark gas is used in the inversion.

diffuse $\mathrm{H}_{2}$ where $\mathrm{CO}$ is disassociated by energetic photons, as well as tracing optically thick $\mathrm{CO}$ and $\mathrm{Hr}$;

- the free-free emission is completely traced by our ionised component and indicates that the free-free estimate from WMAP component separation methods may be too high. In the ionised phase, spinning dust may be present but it is dominated by free-free emission;

- synchrotron emission is well characterised by a power law with a spectral index of -1.0 .

This first analysis of the large-scale Galactic emission as seen by Planck has constrained the large scale ISM properties in the Galaxy. It could be improved in the future via several modifications. Individual regions could be studied using higher angular resolution data, which would probe smaller scale fluctuations that have been missed by the present study. The radial separation could be done differently to explore the difference between arm and inter-arm ISM, in order to focus more on the role of ISM conditions on the star formation process. It should also be possible to obtain an independent estimate of the dark gas in the plane, and ideally some radial information about its behaviour. In the near future, Planck component separation products could be used in the place of WMAP ones. Use of radio recombination line surveys currently underway would be very useful to constrain the free-free emission in the plane, and as a function of Galactic radius.

Acknowledgements. A description of the Planck Collaboration and a list of its members can be found at http://www.rssd.esa.int/index.php? project=PLANCK\&page=Planck_Collaboration 


\section{Appendix A: Table of results}

Table A.1. Result of the inversion per band and per component in $\mathrm{MJy} \mathrm{sr}^{-1} / 10^{20} \mathrm{H}$ atoms, except for the synchrotron component in which case it is in $\mathrm{MJy} \mathrm{sr}^{-1}(v)$ per $\mathrm{MJy} \mathrm{sr}^{-1}(408 \mathrm{MHz})$.

\begin{tabular}{|c|c|c|c|c|c|}
\hline$\underline{\overline{\text { Band [GHz] }}}$ & $\overline{\mathrm{HI}(1)}$ & $\overline{\mathrm{HI}(2)}$ & $\overline{\mathrm{HI}(3)}$ & $\overline{\mathrm{HI}(4)}$ & $\overline{\mathrm{HI}(5)}$ \\
\hline $25000 \ldots \ldots$ & $1.4 \pm 0.4 \times 10^{-1}$ & $5 \pm 3 \times 10^{-2}$ & $7.8 \pm 0.6 \times 10^{-2}$ & $2.4 \pm 0.2 \times 10^{-2}$ & $6.0 \pm 0.9 \times 10^{-3}$ \\
\hline $12000 \ldots \ldots$ & $\times 10^{-1}$ & $\times 10^{-2}$ & $8.2 \pm 1.3 \times 10^{-2}$ & $\times 10^{-2}$ & $\times 10^{-3}$ \\
\hline $5000 \ldots \ldots$ & $8 \pm 3 \times 10^{-1}$ & $3 \pm 2 \times 10^{-1}$ & $4.1 \pm 0.5 \times 10^{-1}$ & $\times 10^{-2}$ & $\times 10^{-2}$ \\
\hline $3000 \ldots \ldots$ & $3.3 \pm 1.5$ & $2 \pm 1$ & $1.8 \pm 0.2$ & $5.9 \pm 0.6 \times 10^{-1}$ & $\times 10^{-2}$ \\
\hline $2143 \ldots \ldots$ & $4.9 \pm 1.2$ & $3.0 \pm 0.8$ & $2.6 \pm 0.2$ & $1.3 \pm 0.1$ & $2.6 \pm 0.3 \times 10^{-1}$ \\
\hline $1250 \ldots \ldots$ & $1.5 \pm 0.6$ & $1.6 \pm 0.4$ & $1.5 \pm 0.1$ & $9.7 \pm 0.4 \times 10^{-1}$ & $4.0 \pm 0.1 \times 10^{-1}$ \\
\hline $857 \ldots \ldots$ & $8 \pm 2 \times 10^{-1}$ & $9.2 \pm 1.7 \times 10^{-1}$ & $7.7 \pm 0.3 \times 10^{-1}$ & $5.7 \pm 0.1 \times 10^{-1}$ & $3.2 \pm 0.1 \times 10^{-1}$ \\
\hline $545 \ldots \ldots$ & $1.9 \pm 0.7 \times 10^{-1}$ & $2.9 \pm 0.5 \times 10^{-1}$ & $2.3 \pm 0.1 \times 10^{-1}$ & $2.0 \pm 0.1 \times 10^{-1}$ & $1.4 \pm 0.1 \times 10^{-1}$ \\
\hline $353 \ldots \ldots$ & $3.8 \pm 1.5 \times 10^{-2}$ & $6.7 \pm 1.2 \times 10^{-2}$ & $5.4 \pm 0.2 \times 10^{-2}$ & $4.9 \pm 0.1 \times 10^{-2}$ & $4.0 \pm 0.1 \times 10^{-2}$ \\
\hline $217 \ldots \ldots$ & $6 \pm 3 \times 10^{-3}$ & $1.4 \pm 0.3 \times 10^{-2}$ & $1.1 \pm 0.1 \times 10^{-2}$ & $1.1 \pm 0.1 \times 10^{-2}$ & $9.5 \pm 0.1 \times 10^{-3}$ \\
\hline $143 \ldots \ldots$ & $1.1 \pm 0.7 \times 10^{-3}$ & $2.7 \pm 0.5 \times 10^{-3}$ & $2.4 \pm 0.1 \times 10^{-3}$ & $2.3 \pm 0.1 \times 10^{-3}$ & $2.3 \pm 0.1 \times 10^{-3}$ \\
\hline $100 \ldots \ldots$ & $<7.3 \quad \times 10^{-4}$ & $6 \pm 2 \times 10^{-4}$ & $5.8 \pm 0.6 \times 10^{-4}$ & $5.9 \pm 0.3 \times 10^{-4}$ & $7.6 \pm 0.1 \times 10^{-4}$ \\
\hline $70 \ldots \ldots$ & $<4.1 \quad \times 10^{-4}$ & $1.1 \pm 0.9 \times 10^{-4}$ & $1.6 \pm 0.5 \times 10^{-4}$ & $1.3 \pm 0.1 \times 10^{-4}$ & $2.9 \pm 0.1 \times 10^{-4}$ \\
\hline $44 \ldots \ldots \ldots$ & $<3.3 \times 10^{-4}$ & $<8.9 \quad \times 10^{-5}$ & $9 \pm 4 \times 10^{-5}$ & $8.3 \pm 1.3 \times 10^{-5}$ & $1.4 \pm 0.2 \times 10^{-4}$ \\
\hline $30 \ldots$ & $<3.4 \quad \times 10^{-4}$ & $1.5 \pm 1.2 \times 10^{-4}$ & $1.3 \pm 0.4 \times 10^{-4}$ & $1.4 \pm 0.1 \times 10^{-4}$ & $1.2 \pm 0.2 \times 10^{-4}$ \\
\hline $1.4 \ldots \ldots$ & $<1.1 \quad \times 10^{-3}$ & $<3.8 \quad \times 10^{-4}$ & $<3.1 \quad \times 10^{-4}$ & $<1.7 \quad \times 10^{-4}$ & $4 \pm 2 \times 10^{-4}$ \\
\hline$\overline{\text { Band [GHz] }}$ & $\mathrm{H}_{2}(1)$ & $\mathrm{H}_{2}(2)$ & $\mathrm{H}_{2}(3)$ & $\mathrm{H}_{2}(4)$ & $\mathrm{H}_{2}(5)$ \\
\hline $25000 \ldots \ldots$ & $2.3 \pm 1.3 \times 10^{-2}$ & $6.2 \pm 0.6 \times 10^{-2}$ & $9 \pm 6 \times 10^{-3}$ & $8 \pm 4 \times 10^{-3}$ & $3.0 \pm 0.6 \times 10^{-2}$ \\
\hline $12000 \ldots \ldots$ & $4.8 \pm 1.9 \times 10^{-2}$ & $5.1 \pm 1.0 \times 10^{-2}$ & $4.8 \pm 1.1 \times 10^{-2}$ & $<1.8 \quad \times 10^{-2}$ & $<3.6 \times 10^{-2}$ \\
\hline $5000 \ldots \ldots$ & $1.7 \pm 1.3 \times 10^{-1}$ & $5.7 \pm 0.7 \times 10^{-1}$ & $2.5 \pm 0.7 \times 10^{-1}$ & $\times 10^{-2}$ & $\times 10^{-1}$ \\
\hline $3000 \ldots \ldots$ & $1.4 \pm 0.6$ & $2.3 \pm 0.3$ & $8 \pm 2 \times 10^{-1}$ & $2.7 \pm 1.0 \times 10^{-1}$ & $9 \pm 2 \times 10^{-1}$ \\
\hline $2143 \ldots \ldots$ & $2.8 \pm 0.4$ & $3.6 \pm 0.2$ & $1.8 \pm 0.2$ & $4.3 \pm 0.9 \times 10^{-1}$ & $1.5 \pm 0.3 \times 10^{0}$ \\
\hline $1250 \ldots \ldots$ & $2.0 \pm 0.2$ & $2.1 \pm 0.1$ & $1.3 \pm 0.1$ & $5.3 \pm 0.4 \times 10^{-1}$ & $1.1 \pm 0.1 \times 10^{0}$ \\
\hline $857 \ldots \ldots$ & $8.0 \pm 0.8 \times 10^{-1}$ & $8.3 \pm 0.4 \times 10^{-1}$ & $6.1 \pm 0.4 \times 10^{-1}$ & $3.8 \pm 0.2 \times 10^{-1}$ & $6.1 \pm 0.5 \times 10^{-1}$ \\
\hline $545 \ldots \ldots$ & $2.1 \pm 0.2 \times 10^{-1}$ & $2.1 \pm 0.1 \times 10^{-1}$ & $1.7 \pm 0.1 \times 10^{-1}$ & $1.4 \pm 0.1 \times 10^{-1}$ & $2.1 \pm 0.1 \times 10^{-1}$ \\
\hline $353 \ldots \ldots$ & $4.3 \pm 0.5 \times 10^{-2}$ & $4.1 \pm 0.3 \times 10^{-2}$ & $3.7 \pm 0.2 \times 10^{-2}$ & $3.7 \pm 0.1 \times 10^{-2}$ & $5.3 \pm 0.3 \times 10^{-2}$ \\
\hline$\ldots \ldots$ & $1.1 \pm 0.1 \times 10^{-2}$ & $1.0 \pm 0.1 \times 10^{-2}$ & $9.5 \pm 0.4 \times 10^{-3}$ & $1.0 \pm 0.1 \times 10^{-2}$ & $1.4 \pm 0.1 \times 10^{-2}$ \\
\hline $143 \ldots \ldots$ & $1.4 \pm 0.2 \times 10^{-3}$ & $1.3 \pm 0.1 \times 10^{-3}$ & $1.2 \pm 0.1 \times 10^{-3}$ & $1.7 \pm 0.1 \times 10^{-3}$ & $2.3 \pm 0.2 \times 10^{-3}$ \\
\hline $100 \ldots \ldots$ & $1.9 \pm 0.1 \times 10^{-3}$ & $1.6 \pm 0.1 \times 10^{-3}$ & $1.5 \pm 0.1 \times 10^{-3}$ & $1.6 \pm 0.1 \times 10^{-3}$ & $1.7 \pm 0.2 \times 10^{-3}$ \\
\hline $70 \ldots \ldots$ & $2.1 \pm 0.5 \times 10^{-4}$ & $1.8 \pm 0.2 \times 10^{-4}$ & $2.2 \pm 0.3 \times 10^{-4}$ & $2.3 \pm 0.1 \times 10^{-4}$ & $3.7 \pm 1.6 \times 10^{-4}$ \\
\hline $44 \ldots \ldots$ & $1.9 \pm 0.4 \times 10^{-4}$ & $1.7 \pm 0.3 \times 10^{-4}$ & $2.4 \pm 0.3 \times 10^{-4}$ & $1.3 \pm 0.1 \times 10^{-4}$ & $4.4 \pm 1.9 \times 10^{-4}$ \\
\hline $30 \ldots \ldots$ & $2.8 \pm 0.4 \times 10^{-4}$ & $2.5 \pm 0.4 \times 10^{-4}$ & $3.1 \pm 0.3 \times 10^{-4}$ & $1.3 \pm 0.1 \times 10^{-4}$ & $6 \pm 2 \times 10^{-4}$ \\
\hline $1.4 \ldots \ldots$ & $<3.6 \quad \times 10^{-4}$ & $\times 10^{-4}$ & $<1.7 \quad \times 10^{-4}$ & $\times 10^{-5}$ & $<5.9 \times 10^{-3}$ \\
\hline$\overline{\text { Band [GHz] }}$. & H II & Sync. & Dark & & \\
\hline $25000 \ldots \ldots$ & $9.7 \pm 0.9 \times 10^{-1}$ & - & $4 \pm 3 \times 10^{-3}$ & & \\
\hline $12000 \ldots \ldots$ & $3.3 \pm 0.3$ & - & $<1.5 \times 10^{-2}$ & & \\
\hline 5000 & $1.9 \pm 0.1 \times 10^{1}$ & - & $\times 10^{-2}$ & & \\
\hline $3000 \ldots \ldots$ & $2.8 \pm 0.1 \times 10^{1}$ & - & $\times 10^{-1}$ & & \\
\hline $2143 \ldots \ldots$ & $2.9 \pm 0.1 \times 10^{1}$ & - & $1.0 \pm 0.1$ & & \\
\hline 1250 & $1.2 \pm 0.1 \times 10^{1}$ & - & $8.3 \pm 0.4 \times 10^{-1}$ & & \\
\hline $857 \ldots \ldots$ & $4.7 \pm 0.2$ & - & $4.4 \pm 0.2 \times 10^{-1}$ & & \\
\hline 545 & $1.3 \pm 0.1$ & - & $1.5 \pm 0.1 \times 10^{-1}$ & & \\
\hline $353 \ldots$ & $3.1 \pm 0.1 \times 10^{-1}$ & - & $3.5 \pm 0.1 \times 10^{-2}$ & & \\
\hline $217 \ldots \ldots$ & $7.9 \pm 0.2 \times 10^{-2}$ & - & $7.9 \pm 0.2 \times 10^{-3}$ & & \\
\hline $143 \ldots$ & $3.0 \pm 0.1 \times 10^{-2}$ & - & $1.8 \pm 0.1 \times 10^{-3}$ & & \\
\hline 100 & $2.3 \pm 0.1 \times 10^{-2}$ & $3 \pm 2 \times 10^{-3}$ & $7.1 \pm 0.3 \times 10^{-4}$ & & \\
\hline 70. & $2.2 \pm 0.1 \times 10^{-2}$ & $\times 10^{-3}$ & $2.5 \pm 0.2 \times 10^{-4}$ & & \\
\hline 44. & $2.3 \pm 0.1 \times 10^{-2}$ & $9 \pm 3 \times 10^{-3}$ & $1.5 \pm 0.2 \times 10^{-4}$ & & \\
\hline 30 . & $2.2 \pm 0.1 \times 10^{-2}$ & $2.0 \pm 0.3 \times 10^{-2}$ & $1.7 \pm 0.2 \times 10^{-4}$ & & \\
\hline $1.4 \ldots \ldots$ & $1.8 \pm 0.1 \times 10^{-2}$ & $2.9 \pm 0.1 \times 10^{-1}$ & $<3.5 \quad \times 10^{-5}$ & & \\
\hline
\end{tabular}

\section{References}

Abdo, A. A., Ackermann, M., Ajello, M., \& Fermi/LAT Collaboration 2010, ApJ, 710, 133

Ackermann, M., Ajello, M., Baldini, L., et al. 2011, ApJ, 726, 81

Ali-Haïmoud, Y., Hirata, C. M., \& Dickinson, C. 2009, MNRAS, 395, 1055

Alves, M. I. R., Davies, R. D., Dickinson, C., et al. 2010, MNRAS, 405, 1654

Banday, A. J., Dickinson, C., Davies, R. D., Davis, R. J., \& Górski, K. M. 2003 , MNRAS, 345, 897

Bennett, C. L., Hill, R. S., Hinshaw, G., et al. 2003, ApJS, 148, 97

Binney, J., \& Merrifield, M. 1998, Galactic astronomy / James Binney and Michael Merrifield. Princeton
Bloemen, J. B. G. M., Strong, A. W., Mayer-Hasselwander, H. A., et al. 1986 A\&A, 154, 25

Bloemen, J. B. G. M., Deul, E. R., \& Thaddeus, P. 1990, A\&A, 233, 437

Boughn, S. P., \& Pober, J. C. 2007, ApJ, 661, 938

Casassus, S., Cabrera, G. F., Förster, F., et al. 2006, ApJ, 639, 951

Casassus, S., Nyman, L.-̊․, Dickinson, C., \& Pearson, T. J. 2007, MNRAS, 382, 1607

Clemens, D. P. 1985, Ph.D. Thesis Massachusetts Univ., 3

Compiègne, M., Verstraete, L., Jones, A., et al. 2011, A\&A, 525, A103

Dame, T. M., Hartmann, D., \& Thaddeus, P. 2001, ApJ, 547, 792

Davies, R. D., Dickinson, C., Banday, A. J., et al. 2006, MNRAS, 370, 1125

Dickey, J. M., McClure-Griffiths, N. M., Gaensler, B. M., \& Green, A. J. 2003, ApJ, 585, 801 
Dickey, J. M., Strasser, S., Gaensler, B. M., et al. 2009, ApJ, 693, 1250 Dickinson, C., Davies, R. D., Bronfman, L., et al. 2007, MNRAS, 379, 297 Dickinson, C., Casassus, S., Davies, R. D., et al. 2010, MNRAS, 407, 2223 Dobler, G., Draine, B., \& Finkbeiner, D. P. 2009, ApJ, 699, 1374 Draine, B. T., \& Lazarian, A. 1998, ApJ, 494, L19

Drimmel, R., Cabrera-Lavers, A., \& López-Corredoira, M. 2003, A\&A, 409, 205 Dunkley, J., Komatsu, E., Nolta, M. R., et al. 2009, ApJS, 180, 306 Eriksen, H. K., Banday, A. J., Górski, K. M., \& Lilje, P. B. 2004, ApJ, 612, 633 Finkbeiner, D. P. 2004, ApJ, 614, 186

Giard, M., Lamarre, J. M., Pajot, F., \& Serra, G. 1994, A\&A, 286, 203 Gold, B., Bennett, C. L., Hill, R. S., et al. 2009, ApJS, 180, 265

Górski, K. M., Hivon, E., Banday, A. J., et al. 2005, ApJ, 622, 759

Grenier, I. A., Casandjian, J.-M., \& Terrier, R. 2005, Science, 307, 1292

Haslam, C. G. T., Salter, C. J., Stoffel, H., \& Wilson, W. E. 1982, A\&AS, 47, 1

Hauser, M. G., Arendt, R. G., Kelsall, T., et al. 1998, ApJ, 508, 25

Heiles, C., \& Troland, T. H. 2003, ApJ, 586, 1067

Jarosik, N., Bennett, C. L., Dunkley, J., et al. 2011, ApJS, 192, 14

Kalberla, P. M. W., Burton, W. B., Hartmann, D., et al. 2005, A\&A, 440, 775

Kogut, A., Banday, A. J., Bennett, C. L., et al. 1996, ApJ, 460, 1

Leach, S. M., Cardoso, J., Baccigalupi, C., et al. 2008, A\&A, 491, 597

Mennella, A., Butler, R. C., Curto, A., et al. 2011, A\&A, 536, A3

Marshall, D. J., Robin, A. C., Reylé, C., Schultheis, M., \& Picaud, S. 2006, A\&A, 453, 635

Mathis, J. S., Mezger, P. G., \& Panagia, N. 1983, A\&A, 128, 212

Miville-Deschênes, M.-A., \& Lagache, G. 2005, ApJS, 157, 302

Miville-Deschênes, M.-A., Ysard, N., Lavabre, A., et al. 2008, A\&A, 490, 1093

Neugebauer, G., Habing, H. J., van Duinen, R., et al. 1984, ApJ, 278, L1

Paladini, R., Montier, L., Giard, M., et al. 2007, A\&A, 465, 839

Planck Collaboration 2011a, A\&A, 536, A1

Planck Collaboration 2011b, A\&A, 536, A2

Planck Collaboration 2011c, A\&A, 536, A7

Planck Collaboration 2011d, A\&A, 536, A8

Planck Collaboration 2011e, A\&A, 536, A9

Planck Collaboration 2011f, A\&A, 536, A10

Planck Collaboration 2011g, A\&A, 536, A11

Planck Collaboration 2011h, A\&A, 536, A12

Planck Collaboration 2011i, A\&A, 536, A13

Planck Collaboration 2011j, A\&A, 536, A14

Planck Collaboration 2011k, A\&A, 536, A15

Planck Collaboration 20111, A\&A, 536, A16

Planck Collaboration 2011m, A\&A, 536, A17

Planck Collaboration 2011n, A\&A, 536, A18

Planck Collaboration 2011o, A\&A, 536, A19

Planck Collaboration 2011p, A\&A, 536, A20

Planck Collaboration 2011q, A\&A, 536, A21

Planck Collaboration 2011r, A\&A, 536, A22

Planck Collaboration 2011s, A\&A, 536, A23

Planck Collaboration 2011t, A\&A, 536, A24

Planck Collaboration 2011u, A\&A, 536, A25

Planck Collaboration 2011v, The Explanatory Supplement to the Planck Early

Release Compact Source Catalogue (ESA)

Planck Collaboration 2011w, A\&A, 536, A26

Planck HFI Core Team 2011a, A\&A, 536, A4

Planck HFI Core Team 2011b, A\&A, 536, A6

Porter, T. A., Moskalenko, I. V., Strong, A. W., Orlando, E., \& Bouchet, L. 2008, ApJ, 682, 400

Press, W. H., Teukolsky, S. A., Vetterling, W. T., \& Flannery, B. P. 1992, Numerical recipes in FORTRAN. The art of scientific computing, ed. W. H. Press, S. A. Teukolsky, W. T. Vetterling, \& B. P. Flannery

Reach, W. T., Koo, B.-C., \& Heiles, C. 1994, ApJ, 429, 672

Reich, P., \& Reich, W. 1986, A\&AS, 63, 205

Reich, P., Testori, J. C., \& Reich, W. 2001, A\&A, 376, 861

Reich, W. 1982, A\&AS, 48, 219

Rouan, D., Leger, A., Omont, A., \& Giard, M. 1992, A\&A, 253, 498

Sale, S. E., Drew, J. E., Unruh, Y. C., et al. 2009, MNRAS, 392, 497

Scaife, A. M. M., Hurley-Walker, N., Davies, M. L., et al. 2008, MNRAS, 385, 809

Scaife, A. M. M., Hurley-Walker, N., Green, D. A., et al. 2009, MNRAS, 400, 1394

Scaife, A. M. M., Nikolic, B., Green, D. A., et al. 2010, MNRAS, 406, L45

Silsbee, K., Ali-Haïmoud, Y., \& Hirata, C. M. 2011, MNRAS, 411, 2750

Sodroski, T. J., Dwek, E., Hauser, M. G., \& Kerr, F. J. 1989, ApJ, 336, 762

Sodroski, T. J., Odegard, N., Arendt, R. G., et al. 1997, ApJ, 480, 173

Tielens, A. G. G. M. 2005, The Physics and Chemistry of the Interstellar Medium, ed. A. G. G. M. Tielens

Watson, R. A., Rebolo, R., Rubiño-Martín, J. A., et al. 2005, ApJ, 624, L89

Weingartner, J. C., \& Draine, B. T. 2001, ApJ, 548, 296

Ysard, N., \& Verstraete, L. 2010, A\&A, 509, A12
Ysard, N., Miville-Deschênes, M. A., \& Verstraete, L. 2010, A\&A, 509, L1

Zacchei, A., Maino, D., Baccigalupi, C., et al. 2011, A\&A, 536, A5

1 Aalto University Metsähovi Radio Observatory, Metsähovintie 114, 02540 Kylmälä, Finland

2 Agenzia Spaziale Italiana Science Data Center, c/o ESRIN, via Galileo Galilei, Frascati, Italy

3 Astroparticule et Cosmologie, CNRS (UMR7164), Université Denis Diderot Paris 7, Bâtiment Condorcet, 10 rue A. Domon et Léonie Duquet, Paris, France

${ }^{4}$ Astrophysics Group, Cavendish Laboratory, University of Cambridge, J J Thomson Avenue, Cambridge CB3 0HE, UK

5 Atacama Large Millimeter/submillimeter Array, ALMA Santiago Central Offices, Alonso de Cordova 3107, Vitacura, Casilla 763 0355, Santiago, Chile

${ }^{6}$ CITA, University of Toronto, 60 St. George St., Toronto, ON M5S 3H8, Canada

7 CNRS, IRAP, 9 Av. colonel Roche, BP 44346, 31028 Toulouse Cedex 4, France

8 California Institute of Technology, Pasadena, California, USA

9 Centre of Mathematics for Applications, University of Oslo, Blindern, Oslo, Norway

10 DAMTP, University of Cambridge, Centre for Mathematical Sciences, Wilberforce Road, Cambridge CB3 OWA, UK

11 DSM/Irfu/SPP, CEA-Saclay, 91191 Gif-sur-Yvette Cedex, France

12 DTU Space, National Space Institute, Juliane Mariesvej 30, Copenhagen, Denmark

13 Departamento de Física, Universidad de Oviedo, Avda. Calvo Sotelo s/n, Oviedo, Spain

14 Department of Astronomy and Astrophysics, University of Toronto, 50 Saint George Street, Toronto, Ontario, Canada

15 Department of Physics \& Astronomy, University of British Columbia, 6224 Agricultural Road, Vancouver, British Columbia, Canada

16 Department of Physics, Gustaf Hällströmin katu 2a, University of Helsinki, Helsinki, Finland

17 Department of Physics, Princeton University, Princeton, New Jersey, USA

18 Department of Physics, Purdue University, 525 Northwestern Avenue, West Lafayette, Indiana, USA

19 Department of Physics, University of California, Berkeley, California, USA

${ }^{20}$ Department of Physics, University of California, One Shields Avenue, Davis, California, USA

21 Department of Physics, University of California, Santa Barbara, California, USA

22 Department of Physics, University of Illinois at Urbana-Champaign, 1110 West Green Street, Urbana, Illinois, USA

23 Dipartimento di Fisica G. Galilei, Università degli Studi di Padova, via Marzolo 8, 35131 Padova, Italy

24 Dipartimento di Fisica, Università La Sapienza, P. le A. Moro 2, Roma, Italy

25 Dipartimento di Fisica, Università degli Studi di Milano, via Celoria 16, Milano, Italy

26 Dipartimento di Fisica, Università degli Studi di Trieste, via A. Valerio 2, Trieste, Italy

27 Dipartimento di Fisica, Università di Ferrara, via Saragat 1, 44122 Ferrara, Italy

28 Dipartimento di Fisica, Università di Roma Tor Vergata, via della Ricerca Scientifica 1, Roma, Italy

29 Discovery Center, Niels Bohr Institute, Blegdamsvej 17, Copenhagen, Denmark

30 Dpto. Astrofísica, Universidad de La Laguna (ULL), 38206 La Laguna, Tenerife, Spain

31 European Southern Observatory, ESO Vitacura, Alonso de Cordova 3107, Vitacura, Casilla 19001, Santiago, Chile

32 European Space Agency, ESAC, Planck Science Office, Camino bajo del Castillo, s/n, Urbanización Villafranca del Castillo, Villanueva de la Cañada, Madrid, Spain 
33 European Space Agency, ESTEC, Keplerlaan 1, 2201 AZ Noordwijk, The Netherlands

${ }^{34}$ Harvard-Smithsonian Center for Astrophysics, 60 Garden Street, Cambridge, MA 02138, USA

35 Helsinki Institute of Physics, Gustaf Hällströmin katu 2, University of Helsinki, Helsinki, Finland

${ }^{36}$ INAF - Osservatorio Astrofisico di Catania, via S. Sofia 78, Catania, Italy

37 INAF - Osservatorio Astronomico di Padova, Vicolo dell'Osservatorio 5, Padova, Italy

38 INAF - Osservatorio Astronomico di Roma, via di Frascati 33, Monte Porzio Catone, Italy

39 INAF - Osservatorio Astronomico di Trieste, via G.B. Tiepolo 11, Trieste, Italy

40 INAF/IASF Bologna, via Gobetti 101, Bologna, Italy

${ }^{41}$ INAF/IASF Milano, via E. Bassini 15, Milano, Italy

${ }^{42}$ INRIA, Laboratoire de Recherche en Informatique, Université Paris-Sud 11, Bâtiment 490, 91405 Orsay Cedex, France

43 IPAG: Institut de Planétologie et d'Astrophysique de Grenoble, Université Joseph Fourier, Grenoble 1 / CNRS-INSU, UMR 5274, 38041 Grenoble, France

44 Imperial College London, Astrophysics group, Blackett Laboratory, Prince Consort Road, London, SW7 2AZ, UK

45 Infrared Processing and Analysis Center, California Institute of Technology, Pasadena, CA 91125, USA

46 Institut Néel, CNRS, Université Joseph Fourier Grenoble I, 25 rue des Martyrs, Grenoble, France

47 Institut d'Astrophysique Spatiale, CNRS (UMR8617) Université Paris-Sud 11, Bâtiment 121, Orsay, France

48 Institut d'Astrophysique de Paris, CNRS UMR7095, Université Pierre \& Marie Curie, 98 bis boulevard Arago, Paris, France

49 Institute of Astronomy and Astrophysics, Academia Sinica, Taipei, Taiwan

50 Institute of Astronomy, University of Cambridge, Madingley Road, Cambridge CB3 OHA, UK

51 Institute of Theoretical Astrophysics, University of Oslo, Blindern, Oslo, Norway

52 Instituto de Astrofísica de Canarias, C/vía Láctea s/n, La Laguna, Tenerife, Spain

53 Instituto de Física de Cantabria (CSIC-Universidad de Cantabria), Avda. de los Castros s/n, Santander, Spain

54 Jet Propulsion Laboratory, California Institute of Technology, 4800 Oak Grove Drive, Pasadena, California, USA

55 Jodrell Bank Centre for Astrophysics, Alan Turing Building, School of Physics and Astronomy, The University of Manchester, Oxford Road, Manchester, M13 9PL, UK

56 Kavli Institute for Cosmology Cambridge, Madingley Road, Cambridge, CB3 OHA, UK
57 LERMA, CNRS, Observatoire de Paris, 61 Avenue de l'Observatoire, Paris, France

${ }^{58}$ Laboratoire AIM, IRFU/Service d'Astrophysique - CEA/DSM CNRS - Université Paris Diderot, Bât. 709, CEA-Saclay, 91191 Gifsur-Yvette Cedex, France

59 Laboratoire Traitement et Communication de l'Information, CNRS (UMR 5141) and Télécom ParisTech, 46 rue Barrault, 75634 Paris Cedex 13, France

${ }^{60}$ Laboratoire de Physique Subatomique et de Cosmologie, CNRS/IN2P3, Université Joseph Fourier Grenoble I, Institut National Polytechnique de Grenoble, 53 rue des Martyrs, 38026 Grenoble Cedex, France

${ }^{61}$ Laboratoire de l'Accélérateur Linéaire, Université Paris-Sud 11, CNRS/IN2P3, Orsay, France

62 Lawrence Berkeley National Laboratory, Berkeley, California, USA

63 Max-Planck-Institut für Astrophysik, Karl-Schwarzschild-Str. 1, 85741 Garching, Germany

64 Max-Planck-Institut für Radioastronomie, Auf dem Hügel 69, 53121 Bonn, Germany

65 MilliLab, VTT Technical Research Centre of Finland, Tietotie 3, Espoo, Finland

66 National University of Ireland, Department of Experimental Physics, Maynooth, Co. Kildare, Ireland

67 Niels Bohr Institute, Blegdamsvej 17, Copenhagen, Denmark

68 Observational Cosmology, Mail Stop 367-17, California Institute of Technology, Pasadena, CA 91125, USA

${ }^{69}$ Optical Science Laboratory, University College London, Gower Street, London, UK

70 SISSA, Astrophysics Sector, via Bonomea 265, 34136 Trieste, Italy

71 SUPA, Institute for Astronomy, University of Edinburgh, Royal Observatory, Blackford Hill, Edinburgh EH9 3HJ, UK

72 School of Physics and Astronomy, Cardiff University, Queens Buildings, The Parade, Cardiff, CF24 3AA, UK

73 Space Sciences Laboratory, University of California, Berkeley, California, USA

74 Spitzer Science Center, 1200 E. California Blvd., Pasadena, California, USA

75 Stanford University, Dept of Physics, Varian Physics Bldg, 382 Via Pueblo Mall, Stanford, California, USA

76 Université de Toulouse, UPS-OMP, IRAP, 31028 Toulouse Cedex 4, France

77 Universities Space Research Association, Stratospheric Observatory for Infrared Astronomy, MS 211-3, Moffett Field, CA 94035, USA

78 University of Granada, Departamento de Física Teórica y del Cosmos, Facultad de Ciencias, Granada, Spain

79 University of Miami, Knight Physics Building, 1320 Campo Sano Dr., Coral Gables, Florida, USA

80 Warsaw University Observatory, Aleje Ujazdowskie 4, 00-478 Warszawa, Poland 